\title{
Primeiro levantamento da fauna de Ephemeroptera (Insecta) do Espírito Santo, Sudeste do Brasil
}

\author{
Frederico Falcão Salles ${ }^{1,3}$, Jeane Marcelle Cavalcante do Nascimento ${ }^{1}$, Fabiana Criste Massariol, \\ Kamila Batista Angeli ${ }^{1}$ Patrik Barcelos e Silva ${ }^{1}$, Jéssika Ana Rúdio ${ }^{1}$ \& Rafael Boldrini ${ }^{2}$ \\ ${ }^{1}$ Departamento de Ciências Agrárias e Biológicas, Centro Universitário Norte do Espírito Santo, \\ Universidade Federal do Espírito Santo - UFES, \\ CEP 29932-540 São Mateus, ES, Brasil \\ ${ }^{2}$ Divisão de Curso de Entomologia - DCEN, Instituto Nacional de Pesquisas da Amazônia - INPA, \\ CEP 69011-970 Manaus, AM, Brasil \\ ${ }^{3}$ Autor para correspondencia: Frederico Falcão Salles, e-mail: ffsalles@gmail.com
}

SALLES, F.F., NASCIMENTO, J., MASSARIOL, F., ANGELI, K., BARCELOS-SILVA, P., RÚDIO, J. \& BOLDRINI, R. First survey of mayflies (Ephemeroptera, Insecta) from Espírito Santo State, Southeastern Brazil. Biota Neotrop. 10(1): http://www.biotaneotropica.org.br/v10n1/en/abstract?inventory+bn02610012010.

Abstract: Based on collections performed between 2008 and 2009 at Parque Nacional do Caparaó, Reserva Biológica de Sooretama, among other areas in Espírito Santo State, southeastern Brazil, we present the first survey of mayflies (Insecta: Ephemeroptera) from the state. Despite the fact that the state is one of the smallest in Brazil, representing only $0.53 \%$ of the country area, a surprising diversity was found. Seventy-six species, 41 genera and nine families were identified, which represents about $25 \%$ of the species reported from Brazil, $65 \%$ of the genera, and almost all families. Of these, 17 species (most of Baetidae and Leptophlebiidae) are new to science. Five species, collected only at the nymphal stage, could not be identified to the species level. The present work demonstrates that, as for other organisms, the diversity of mayflies is high in the state, and more works aiming to investigate the aquatic insect composition of the Espírito Santo State should be encouraged.

Keywords: aquatic insects, diversity, neotropical, Caparaó, Sooretama.

SALlES, F.F., NASCIMENTO, J., MASSARIOL, F., ANGELI, K., BARCELOS-SILVA, P., RÚDIO, J. \& BOLDRINI, R. Primeiro levantamento da fauna de Ephemeroptera (Insecta) do Espírito Santo, Sudeste do Brasil. Biota Neotrop. 10(1): http://www.biotaneotropica.org.br/v10n1/pt/abstract?inventory+bn02610012010.

Resumo: A partir de coletas realizadas entre 2008 e 2009 no Parque Nacional do Caparaó, Reserva Biológica de Sooretama, entre outras áreas do Espírito Santo, apresentamos o primeiro levantamento da fauna de Ephemeroptera do estado. A despeito do seu pequeno tamanho, pois a área do estado representa apenas $0,53 \%$ do território nacional, uma surpreendente diversidade de Ephemeroptera foi encontrada. Ao todo foram identificadas 76 espécies, 41 gêneros e nove famílias, que representam aproximadamente $25 \%$ das espécies brasileiras, $65 \%$ dos gêneros e quase todas as famílias ocorrentes no Brasil. Destes táxons, 17 espécies (a maioria de Baetidae e Leptophlebiidae) são novos para a ciência. Cinco espécies, coletadas apenas no estágio ninfal, não puderam ser identificadas. O presente trabalho demonstra que, como para muitos organismos, a diversidade de Ephemeroptera para o estado é alta e mais pesquisas sobre a composição faunística de insetos aquáticos no Espírito Santo devem ser incentivadas.

Palavras-chave: insetos aquáticos, diversidade, neotrópico, Caparaó, Sooretama. 


\section{Introdução}

O conhecimento acerca dos insetos aquáticos do Estado do Espírito Santo, apesar de este estar localizado numa das regiões que abriga grande parte dos estudos a respeito dessa fauna no Brasil, pode ser considerado incipiente e fragmentado. Com exceção da ordem Odonata, onde diversos trabalhos abordaram especificamente a sua fauna (e.g. Costa \& Oldrini 2005), poucos esforços foram realizados no sentido de se compreender a diversidade dos insetos aquáticos na área. Tal situação num estado que, como o Espírito Santo, está localizado numa área de alto grau de endemismo e diversidade biológica (IPEMA 2007) e vem sofrendo fortes pressões antrópicas nas últimas décadas, dificulta ou mesmo impede qualquer esforço prático para o estabelecimento de áreas ou habitats aquáticos prioritários para a conservação (Lugo-Ortiz et al. 2002).

Com base na publicação da Lista das espécies de Ephemeroptera registradas para o Brasil (Salles et al. 2004), a qual vem sendo atualizada freqüentemente pela internet no sítio Ephemeroptera do Brasil (http://sites.google.com/site/ephemeropterabr/home), o Espírito Santo foi considerado um dos estados brasileiros menos estudados com relação ao grupo no país, encontrando-se numa situação comparável apenas aos estados da Região Nordeste bem como à maioria da Região Norte. Até 2008, apenas 12 espécies haviam sido registradas para o Espírito Santo, sendo praticamente metade dessas baseadas em apenas uma coleta na Reserva Santa Lúcia, município de Santa Teresa (Lugo-Ortiz et al. 2002, Salles 2009).

A partir de então uma série de projetos com objetivos de incrementar o conhecimento a respeito dos insetos aquáticos do estado começou a ser realizada, tanto em áreas de conservação, como o Parque Nacional do Caparaó, as Reservas Biológicas de Sooretama, Augusto Ruschi e Córrego do Veado, bem como algumas áreas particulares. Como resultado, alguns trabalhos taxonômicos abordando a descrição de inúmeras novas espécies ou novos registros foram publicados ou recentemente submetidos [i.e., Salles \& Boldrini (2008), Boldrini \& Salles (2009), Cruz et al. (2009), Salles \& Nascimento (2009), Boldrini et al. (no prelo a, b), De Souza et al. (no prelo), Salles et al. (dados não publicados)]. Em função da grande diversidade encontrada, da urgência em se conhecer melhor a fauna de Ephemeroptera e insetos aquáticos do estado, bem como do longo tempo necessário até que trabalhos taxonômicos sejam preparados para os diferentes grupos encontrados, optamos por apresentar o presente trabalho. $\mathrm{O}$ principal objetivo deste trabalho é apresentar uma lista dos gêneros e espécies de Ephemeroptera encontrados até o momento para o Espírito Santo.

\section{Materiais e Métodos}

\section{1. Áreas de estudo}

O Espírito Santo, com uma área de $45.597 \mathrm{~km}^{2}$, está localizado na Região Sudeste do Brasil, entre os paralelos $17^{\circ} 53$ ' 29"' S e $21^{\circ} 18^{\prime} 03^{\prime \prime} \mathrm{S}$ e os meridianos $39^{\circ} 41^{\prime} 18^{\prime \prime} \mathrm{O}$ e $41^{\circ} 25^{\prime} 45^{\prime \prime} \mathrm{O}$. O estado possuía quase $90 \%$ da sua superfície coberta por Mata Atlântica, sendo o restante ocupado por ecossistemas associados, como brejos, restingas, mangues, campos de altitude e campos rupestres. O relevo caracteriza-se como montanhoso, com altitudes variando desde o nível do mar a $2.897 \mathrm{~m}$, enquanto o clima predominante é o tropical, quente e úmido no litoral e temperado na região serrana (IPEMA 2007).

Com o objetivo de abranger a maior diversidade possível de ecossistemas no estado, e conseqüentemente da fauna de Ephemeroptera, optamos por priorizar áreas localizadas em distintas regiões e bacias hidrográficas (Figura 1). Dentre as áreas mais estudadas destacam-se o Parque Nacional do Caparaó e a Reserva Biológica de Sooretama (maiores informações abaixo). Entretanto, coletas esporádicas também foram feitas nas Reservas Biológicas Augusto Ruschi e Córrego do Veado, bem como em áreas particulares nos municípios de Santa Teresa, Santa Leopoldina, Alfredo Chaves, Águia Branca, Linhares, São Mateus e Conceição da Barra (Tabela 1).

O Parque Nacional do Caparaó (Figura 2) possui 31.853 ha e está localizado no sudoeste do estado, na divisa com Minas Gerais, entre as coordenadas geográficas $20^{\circ} 19^{\prime}-20^{\circ} 37^{\prime} \mathrm{S}$ e $41^{\circ} 43^{\prime}-4^{\circ} 53^{\prime} \mathrm{O}$. Cerca de $60 \%$ de sua área, ou aproximadamente 18.000 ha, está localizada no Espírito Santo. Apresenta vegetação caracterizada como Floresta Ombrófila Densa Altimontana e Floresta Estacional Semidecidual, além de preservar o único local de ocorrência de campos de altitude no Estado. As altitudes no parque variam de 997 a 2.897 m acima do nível do mar (IPEMA 2007). A rede de drenagem é

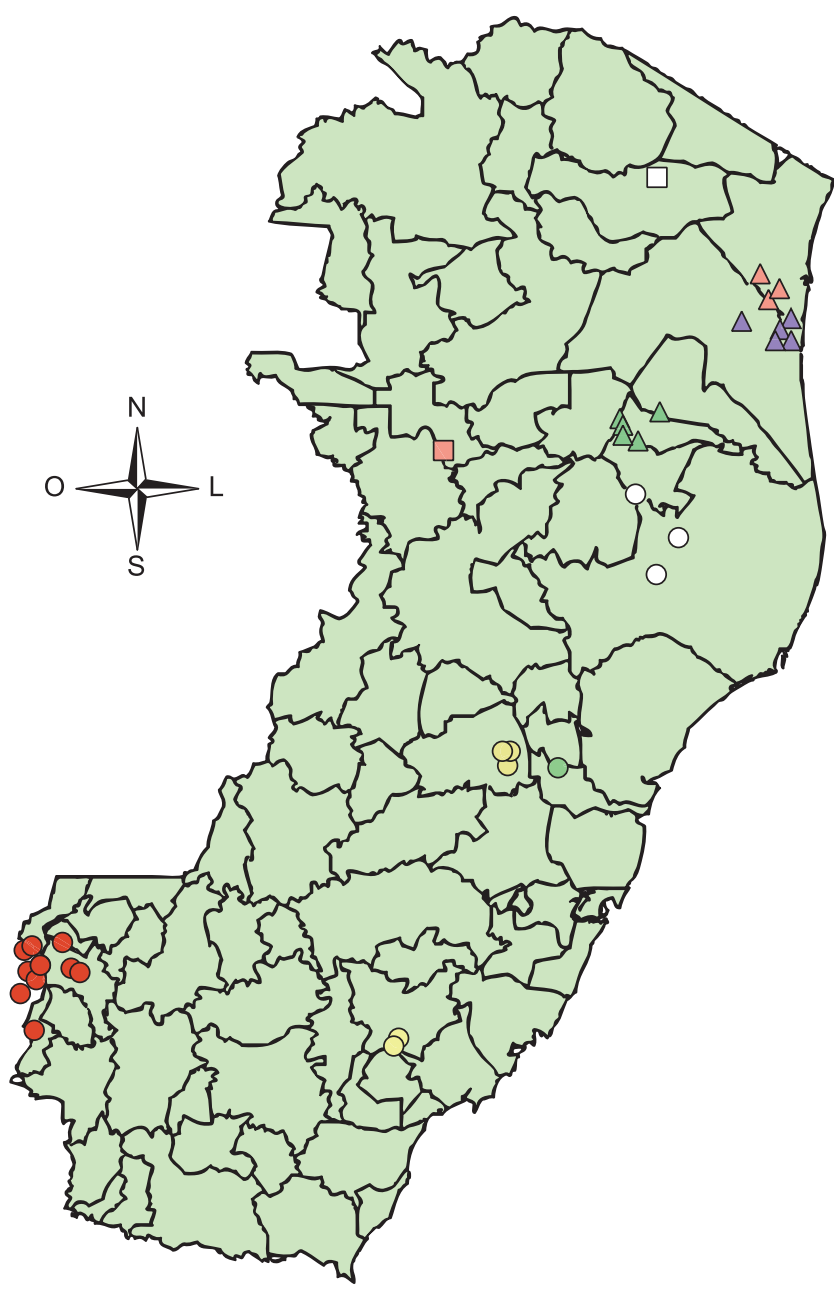

Figura 1. Mapa do Estado do Espírito Santo mostrando a distribuição dos pontos de coleta. Círculos vermelhos = Parque Nacional do Caparaó. Círculos amarelos = Alfredo Chaves. Círculos laranjas = Santa Teresa . Círculo verde $=$ Fundão. Círculos brancos $=$ lagoas de Linhares e Sooretama. Triângulos verdes = Reserva Biológica de Sooretama. Quadrado vermelho = Águia Branca. Triângulos azuis $=$ São Mateus. Triângulos vermelhos $=$ Conceição da Barra. Quadrado branco $=$ Reserva Biológica do Córrego do Veado.

Figure 1. Map of Espírito Santo State showing the distribution of collection stations. Red circles $=$ Parque Nacional do Caparaó. Yellow circles $=$ Alfredo Chaves . Orange circles $=$ Santa Teresa . Green circle $=$ Fundão. White circles $=$ lagoons from Linhares and Sooretama. Green triangles $=$ Reserva Biológica de Sooretama. Red square = Águia Branca. Blue triangles = São Mateus. Red triangles = Conceição da Barra. White square $=$ Reserva Biológica do Córrego do Veado. 
Ephemeroptera do Estado do Espírito Santo

Tabela 1. Pontos de coletas no Espírito Santo e na divisa com Minas Gerais (município de Espera Feliz e Caparaó), acompanhados pelo município, localidade, coordenadas geográficas e altitude.

Table 1. Sample sites at Espírito Santo State and at its border with Minas Gerais State (Espera Feliz city and Caparaó city), followed by municipality, locality, geographic coordinates, and altitudes.

\begin{tabular}{|c|c|c|c|c|}
\hline Ponto & Município & Localidade & Coordenadas & Altitude \\
\hline PT 01 & Águia Branca & Pedra Torta & $19^{\circ} 3^{\prime} 56^{\prime \prime} \mathrm{S}$ e $40^{\circ} 42^{\prime} 45,3^{\prime \prime} \mathrm{W}$ & $156 \mathrm{~m}$ \\
\hline PT 02 & Alfredo Chaves & $\begin{array}{l}\text { Nova Mantova. Afluente da margem esquerda do } \\
\text { córrego }\end{array}$ & $20^{\circ} 39^{\prime} 45,9^{\prime \prime} \mathrm{S}$ e $40^{\circ} 50^{\prime} 43,6^{\prime \prime} \mathrm{W}$ & $472 \mathrm{~m}$ \\
\hline PT 03 & Alfredo Chaves & Nova Mantova. Afluente da margem direita do córrego & $20^{\circ} 40^{\prime} 06,6^{\prime \prime} \mathrm{S}$ e $40^{\circ} 50^{\prime} 36,1^{\prime \prime} \mathrm{W}$ & $544 \mathrm{~m}$ \\
\hline PT 04 & Alfredo Chaves & Nova Mantova. Fazenda Nego Boldrini, córrego & $20^{\circ} 39^{\prime} 25^{\prime \prime} \mathrm{S}$ e $40^{\circ} 50^{\prime} 14.6^{\prime \prime} \mathrm{W}$ & $371 \mathrm{~m}$ \\
\hline PT 05 & Alto Caparaó & Queda d'água Casa Queimada & $20^{\circ} 27^{\prime} 21.4^{\prime \prime} \mathrm{S}$ e $41^{\circ} 48^{\prime} 32.1^{\prime \prime} \mathrm{W}$ & $2250 \mathrm{~m}$ \\
\hline PT 06 & Alto Caparaó & Córrego entre Casa Queimada e Cachoeira da Farofa & $20^{\circ} 27^{\prime} 45.8^{\prime \prime} \mathrm{S}$ e $41^{\circ} 48^{\prime} 24.7^{\prime \prime} \mathrm{W}$ & $2160 \mathrm{~m}$ \\
\hline PT 07 & Alto Caparaó & Rio São Domingos, Cachoeira da Farofa & $20^{\circ} 28^{\prime} 19.5^{\prime \prime} \mathrm{S}$ e $41^{\circ} 49^{\prime} 41.7^{\prime \prime} \mathrm{W}$ & $1972 \mathrm{~m}$ \\
\hline PT 08 & Alto Caparaó & Vale Verde & $20^{\circ} 25^{\prime} 11,6^{\prime \prime} \mathrm{S}$ e $41^{\circ} 50^{\prime} 45.7^{\prime \prime} \mathrm{W}$ & $1309 \mathrm{~m}$ \\
\hline PT 09 & Caparaó & Fazenda Casa do Lajeado, Cachoeira do Cambucá & $20^{\circ} 37^{\prime} 28.2^{\prime \prime} \mathrm{S}$ e $41^{\circ} 49^{\prime} 26.0^{\prime \prime} \mathrm{W}$ & $893 \mathrm{~m}$ \\
\hline PT 10 & Caparaó & Vale Encantado & $20^{\circ} 24^{\prime} 38,7^{\prime \prime} \mathrm{S}$ e $41^{\circ} 50^{\prime} 03.6^{\prime \prime} \mathrm{W}$ & $1976 \mathrm{~m}$ \\
\hline PT 11 & Espera Feliz & Cachoeira Vale a Pena & $20^{\circ} 32^{\prime} 19.6^{\prime \prime} \mathrm{S}$ e $41^{\circ} 51^{\prime} 25.2^{\prime \prime} \mathrm{W}$ & $1053 \mathrm{~m}$ \\
\hline PT 12 & Espera Feliz & Córrego da Calha & $20^{\circ} 29^{\prime} 26.0^{\prime \prime} \mathrm{S}$ e $41^{\circ} 49^{\prime} 17.5^{\prime \prime} \mathrm{W}$ & $1612 \mathrm{~m}$ \\
\hline PT 13 & Espera Feliz & Córrego do Batista & $20^{\circ} 30^{\prime} 07.1^{\prime \prime} \mathrm{S}$ e $41^{\circ} 49^{\prime} 09.8^{\prime \prime} \mathrm{W}$ & $1411 \mathrm{~m}$ \\
\hline PT 14 & Espera Feliz & Macieira, Brejo & $20^{\circ} 28^{\prime} 51.8^{\prime \prime} \mathrm{S}$ e $41^{\circ} 49^{\prime} 43.5^{\prime \prime} \mathrm{W}$ & $1857 \mathrm{~m}$ \\
\hline PT 15 & Espera Feliz & Rio Preto & $20^{\circ} 30^{\prime} 08.9^{\prime \prime} \mathrm{S}$ e $41^{\circ} 49^{\prime} 13.4^{\prime \prime} \mathrm{W}$ & $822 \mathrm{~m}$ \\
\hline PT 16 & Espera Feliz & Rio São Domingos, Cachoeira do Aurélio & $20^{\circ} 28^{\prime} 57.5^{\prime \prime} \mathrm{S}$ e $41^{\circ} 50^{\prime} 14.5^{\prime \prime} \mathrm{W}$ & $1800 \mathrm{~m}$ \\
\hline PT 17 & Espera Feliz & Rio São Domingos, Macieira & $20^{\circ} 28^{\prime} 52.6^{\prime \prime} \mathrm{S}$ e $41^{\circ} 49^{\prime} 44.6^{\prime \prime} \mathrm{W}$ & $1854 \mathrm{~m}$ \\
\hline PT 18 & Espera Feliz & Rio São Domingos, Macieira & $20^{\circ} 28^{\prime} 57.1^{\prime \prime} \mathrm{S}$ e $41^{\circ} 49^{\prime} 50.4^{\prime \prime} \mathrm{W}$ & $1850 \mathrm{~m}$ \\
\hline PT 19 & Ibitirama & Rio do Tecnotruta, propriedade "Sonho Meu" & $20^{\circ} 28^{\prime} 08.8^{\prime \prime} \mathrm{S}$ e $41^{\circ} 43^{\prime} 22.5^{\prime \prime} \mathrm{W}$ & $959 \mathrm{~m}$ \\
\hline PT 20 & Ibitirama & Rio entre Pedra Roxa e Santa Marta & $20^{\circ} 28^{\prime} 43.0^{\prime \prime} \mathrm{S}$ e $41^{\circ} 42^{\prime} 15.8^{\prime} \mathrm{W}$ & $833 \mathrm{~m}$ \\
\hline PT 21 & Ibitirama & Afulente do Rio Pedra Roxa & $20^{\circ} 23^{\prime} 48.1^{\prime \prime} \mathrm{S}$ e $41^{\circ} 44^{\prime} 08.1^{\prime \prime} \mathrm{W}$ & $1063 \mathrm{~m}$ \\
\hline PT 22 & Ibitirama & Rio Pedra Roxa, sede do Ibama & $20^{\circ} 23^{\prime} 48.1^{\prime \prime} \mathrm{S}$ e $41^{\circ} 44^{\prime} 08.1^{\prime \prime} \mathrm{W}$ & $1063 \mathrm{~m}$ \\
\hline PT 23 & Ibitirama & Rio Pedra Roxa, Vertente da Pedra Roxa, Sr. Menário & $20^{\circ} 24^{\prime} 20.7^{\prime \prime} \mathrm{S}$ e $41^{\circ} 43^{\prime} 35.6^{\prime \prime} \mathrm{W}$ & 997 m \\
\hline PT 24 & Iúna & Córrego na estrada & $20^{\circ} 24^{\prime} 05.5^{\prime \prime} \mathrm{S}$ e $41^{\circ} 43^{\prime} 45.9^{\prime \prime} \mathrm{W}$ & $1015 \mathrm{~m}$ \\
\hline PT 25 & Conceição da Barra & $\begin{array}{l}\text { Primeiro ponto na estrada de chão de São Mateus para } \\
\text { Conceição da Barra }\end{array}$ & $18^{\circ} 38^{\prime} 02,5^{\prime \prime} \mathrm{S}$ e $39^{\circ} 48^{\prime} 50,4^{\prime \prime} \mathrm{W}$ & $21 \mathrm{~m}$ \\
\hline PT 26 & Conceição da Barra & $\begin{array}{l}\text { Segundo ponto na estrada de chão de São Mateus para } \\
\text { Conceição da Barra }\end{array}$ & $18^{\circ} 39^{\prime} 21,9^{\prime \prime} \mathrm{S}$ e $39^{\circ} 50^{\prime} 06,1^{\prime \prime} \mathrm{W}$ & $19 \mathrm{~m}$ \\
\hline PT 27 & Conceição da Barra & $\begin{array}{l}\text { Terceiro ponto na estrada de chão de São Mateus para } \\
\text { Conceição da Barra }\end{array}$ & $18^{\circ} 35^{\prime} 18,3^{\prime \prime} \mathrm{S}$ e $39^{\circ} 51^{\prime} 37,9^{\prime \prime} \mathrm{W}$ & $26 \mathrm{~m}$ \\
\hline PT 28 & Linhares & Lagoa Nova & $19^{\circ} 25^{\prime} 07.2^{\prime \prime} \mathrm{S}$ e $40^{\circ} 09^{\prime} 26.4^{\prime \prime} \mathrm{W}$ & $17 \mathrm{~m}$ \\
\hline PT 29 & Linhares & Lagoa Juparanã - Praia do Minotauro & $19^{\circ} 19^{\prime} 5,8^{\prime \prime} \mathrm{S}$ e $40^{\circ} 05^{\prime} 11,9^{\prime \prime} \mathrm{W}$ & $17 \mathrm{~m}$ \\
\hline PT 30 & São Mateus & Pesque-pague Cantinho de Maria & $18^{\circ} 42^{\prime} 36.0^{\prime \prime} \mathrm{S}$ e $39^{\circ} 46^{\prime} 48.0^{\prime \prime} \mathrm{W}$ & $2 \mathrm{~m}$ \\
\hline PT 31 & São Mateus & Carvoaria & $18^{\circ} 46^{\prime} 04.0^{\prime \prime} \mathrm{S}$ e $39^{\circ} 49^{\prime} 06.0^{\prime \prime} \mathrm{W}$ & $18 \mathrm{~m}$ \\
\hline PT 32 & São Mateus & Rio Preto & $18^{\circ} 44^{\prime} 08.0^{\prime \prime} \mathrm{S}$ e $39^{\circ} 47^{\prime} 47.0^{\prime \prime} \mathrm{W}$ & $6 \mathrm{~m}$ \\
\hline PT 33 & São Mateus & Grande Alagado & $18^{\circ} 46^{\prime} 17.0^{\prime \prime} \mathrm{S}$ e $39^{\circ} 48^{\prime} 55.0^{\prime \prime} \mathrm{W}$ & $13 \mathrm{~m}$ \\
\hline PT 34 & São Mateus & Sítio São Lázaro & $18^{\circ} 43^{\prime} 23.0^{\prime \prime} \mathrm{S}$ e $39^{\circ} 54^{\prime} 42.0^{\prime \prime} \mathrm{W}$ & $35 \mathrm{~m}$ \\
\hline PT 35 & São Mateus & Poças do Bairro Liberdade & $18^{\circ} 44^{\prime} 33.4^{\prime \prime} \mathrm{S}$ e $39^{\circ} 48^{\prime} 38.1^{\prime \prime} \mathrm{W}$ & $13 \mathrm{~m}$ \\
\hline PT 36 & São Mateus & Represa na estrada para Barra Nova & $18^{\circ} 46^{\prime} 27.57^{\prime \prime} \mathrm{S}$ e $39^{\circ} 46^{\prime} 41.96^{\prime \prime} \mathrm{W}$ & $4 \mathrm{~m}$ \\
\hline PT 37 & Sooretama & Rio Barra Seca & $18^{\circ} 57^{\prime} 52.1^{\prime \prime} \mathrm{S}$ e $40^{\circ} 07^{\prime} 37.3^{\prime \prime} \mathrm{W}$ & $5 \mathrm{~m}$ \\
\hline
\end{tabular}


Tabela 1. Continuação

\begin{tabular}{|c|c|c|c|c|}
\hline Ponto & Município & Localidade & Coordenadas & Altitude \\
\hline PT 38 & Sooretama & Cachoeira Bomjardim & $18^{\circ} 59^{\prime} 56.5^{\prime \prime} \mathrm{S}$ e $40^{\circ} 14^{\prime} 04.0^{\prime \prime} \mathrm{W}$ & $39 \mathrm{~m}$ \\
\hline PT 39 & Sooretama & Córrego Quirinho, à jusante & $19^{\circ} 02^{\prime} 72.2^{\prime \prime} \mathrm{S}$ e $40^{\circ} 10^{\prime} 68.0^{\prime \prime} \mathrm{W}$ & $69 \mathrm{~m}$ \\
\hline PT 40 & Sooretama & Rio Paraisópolis & $18^{\circ} 59^{\prime} 03.0^{\prime \prime} \mathrm{S}$ e $40^{\circ} 14^{\prime} 32.3$ ' W & $38 \mathrm{~m}$ \\
\hline PT 41 & Sooretama & Córrego Quirininho, à montante & $19^{\circ} 02^{\prime} 43.1^{\prime \prime} \mathrm{S}$ e $40^{\circ} 10^{\prime} 40.7 ” \mathrm{~W}$ & $62 \mathrm{~m}$ \\
\hline PT 42 & Sooretama & Córrego Rodrigues & $19^{\circ} 01^{\prime} 36.6^{\prime \prime} \mathrm{S}$ e $40^{\circ} 13^{\prime} 39^{\prime \prime} \mathrm{W}$ & $44 \mathrm{~m}$ \\
\hline PT 43 & Santa Teresa & $\begin{array}{l}\text { Nova Lombardia, Capitel de Santo Antônio, Cachoeira } \\
\text { Grande }\end{array}$ & $19^{\circ} 52^{\prime} 30.8^{\prime \prime} \mathrm{S}$ e $40^{\circ} 31^{\prime} 49.1^{\prime \prime} \mathrm{W}$ & $712 \mathrm{~m}$ \\
\hline PT 44 & Santa Teresa & $\begin{array}{l}\text { Nova Lombardia, Capitel de Santo Antônio, Córrego } \\
\text { Escavado }\end{array}$ & $19^{\circ} 31^{\prime} 47.3^{\prime \prime} \mathrm{S}$ e $40^{\circ} 31^{\prime} 47.3$ ” W & $705 \mathrm{~m}$ \\
\hline PT 45 & Santa Teresa & $\begin{array}{l}\text { Nova Lombardia, Capitel de Santo Antônio, Córrego } \\
\text { Grande }\end{array}$ & $19^{\circ} 52^{\prime} 31.6^{\prime \prime} \mathrm{S}$ e $40^{\circ} 31^{\prime} 49.1^{\prime \prime} \mathrm{W}$ & $768 \mathrm{~m}$ \\
\hline PT 46 & Santa Teresa & Nova Lombardia, Poção & $19^{\circ} 52^{\prime} 30.9^{\prime \prime} \mathrm{S}$ e $40^{\circ} 32^{\prime} 07.4^{\prime \prime} \mathrm{W}$ & $739 \mathrm{~m}$ \\
\hline PT 47 & Santa Teresa & Rebio Augusto Ruschi, Cachoeira Grande & $19^{\circ} 52^{\prime} 30.1^{\prime \prime} \mathrm{S}$ e $40^{\circ} 33^{\prime} 21.9^{\prime \prime} \mathrm{W}$ & $704 \mathrm{~m}$ \\
\hline PT 48 & Santa Teresa & Rebio Augusto Ruschi, Cachoeira do Meio & $19^{\circ} 53^{\prime} 20.6^{\prime \prime} \mathrm{S}$ e $40^{\circ} 32^{\prime} 41.5^{\prime \prime} \mathrm{W}$ & $803 \mathrm{~m}$ \\
\hline PT 49 & Santa Teresa & Rebio Augusto Ruschi, afluente & $19^{\circ} 55^{\prime} 22.5^{\prime \prime} \mathrm{S}$ e $40^{\circ} 33^{\prime} 13.4^{\prime \prime} \mathrm{W}$ & $805 \mathrm{~m}$ \\
\hline PT 50 & Fundão & Timbuí, Hotel Fazenda Lua Nova & $19^{\circ} 56^{\prime} 02^{\prime \prime} \mathrm{S}$ e $40^{\circ} 24^{\prime} 45^{\prime \prime} \mathrm{W}$ & $57 \mathrm{~m}$ \\
\hline PT 51 & Pinheiros & Rebio Córrego do Veado, Rio Sede & $18^{\circ} 22^{\prime} 16,8^{\prime \prime} \mathrm{S}$ e $40^{\circ} 8{ }^{\prime} 30,2^{\prime \prime} \mathrm{W}$ & $55 \mathrm{~m}$ \\
\hline PT 52 & Pinheiros & Rebio Córrego do Veado, Água Limpa & $18^{\circ} 22^{\prime} 4,1^{\prime \prime} \mathrm{S}$ e $40^{\circ} 8^{\prime} 23,8^{\prime \prime} \mathrm{W}$ & $41 \mathrm{~m}$ \\
\hline PT 53 & Alto Caparaó & $\begin{array}{l}\text { Córrego Casa Queimada (córrego que passa ao lado } \\
\text { da casa) }\end{array}$ & $20^{\circ} 27^{\prime} 29,4^{\prime \prime} \mathrm{S}$ e $41^{\circ} 48^{\prime} 30,3^{\prime \prime} \mathrm{W}$ & $2188 \mathrm{~m}$ \\
\hline PT 54 & Sooretama & Patrimônio da Lagoa & $19^{\circ} 10^{\prime} 09,7^{\prime \prime} \mathrm{S}$ e $40^{\circ} 11^{\prime} 25,2^{\prime \prime}$ & $20 \mathrm{~m}$ \\
\hline
\end{tabular}

caracterizada por numerosos rios perenes, de pequeno e médio porte, com forte declividade, sendo freqüente a ocorrência de corredeiras, rápidos e algumas cachoeiras (IBAMA 2007).

A Reserva Biológica de Sooretama (Figura 3) possui 24.250 ha e está localizada no município de Linhares, entre as coordenadas $18^{\circ} 33^{\prime}-19^{\circ} 05^{\prime} \mathrm{S}$ e $39^{\circ} 55^{\prime}-40^{\circ} 15^{\prime}$ O. É constituída, predominantemente, por Floresta Ombrófila Densa de Terras Baixas, mais conhecida como "Mata de Tabuleiros". Esta unidade tem área contígua à Reserva Natural do Vale do Rio Doce e juntas constituem o maior maciço florestal do Estado do Espírito Santo, favorecendo a presença de uma valiosa diversidade florística e faunística (IPEMA 2007). A área da reserva é delimitada ao norte pelo Rio Barra Seca e ao sul pelo córrego Cupido. Diversos córregos menores, afluentes do Barra Seca ou do Cupido, cortam a reserva e apresentam nascentes tanto dentro quanto fora de sua área.

\section{Coletas, identificações e deposição}

As coletas, sempre qualitativas, foram realizadas basicamente de duas maneiras: com uso de peneiras e puçás com abertura de malha de aproximadamente $1,0 \mathrm{~mm}$, no caso das ninfas; e a partir de armadilhas luminosas do tipo lençol ou Pensilvânia, no caso dos alados. Redes aéreas também foram utilizadas para a coleta de alados que eventualmente estivessem revoando. Durante a coleta das ninfas, os indivíduos de cada meso-habitat eram acondicionados em potes individualizados e devidamente etiquetados. Os meso-habitats foram definidos como areia $(0,05-2,00 \mathrm{~mm})$, cascalho $(2,00 \mathrm{~mm}-2,00 \mathrm{~cm})$, pedra rolada $(2,00-20,00 \mathrm{~cm})$, matacão $(>20 \mathrm{~cm})$, laje, folhiço de superfície (folhas e troncos presos na superfície da água, geralmente em áreas de correnteza moderada a forte), folhiço de fundo (folhas e troncos no leito dos corpos d'água, em áreas de remanso), vegeta- ção marginal (vegetação terrestre presente nas margens dos corpos d'água, mas que em geral possuem algumas partes em contato com a água) e macrófitas. Nos ambientes lóticos, informações referentes à velocidade da água também foram anotadas, sendo consideradas as categorias, sem correnteza, correnteza fraca, moderada, forte e muito forte.

Como a taxonomia de muitos representantes da ordem se baseia no estágio adulto (imago), ninfas de último estádio, evidenciadas pela coloração negra das tecas alares anteriores, foram criadas em campo. As criações foram realizadas colocando-se as ninfas em copos plásticos, telados no fundo e cobertos com um filó na boca, arranjados em orifícios sobre uma placa de isopor que por sua vez ficava flutuando sobre a água e presa à margem. Como os representantes da ordem passam por um estágio alado antes de se tornarem propriamente adultos, conhecido como subimago, sempre que obtidas, por meio de criação ou armadilha, subimagos eram acondicionadas em um pote plástico, de aproximadamente $15 \mathrm{ml}$, até que fizessem a última muda. Em seguida, aguardávamos de 12 a 24 horas para fixá-las, procedimento importante para conservação da coloração.

As identificações foram realizadas com base em Domínguez et al. (2006) e Salles (2006), assistidas, sempre que necessário, por artigos pertinentes a cada táxon. Grande parte da bibliografia a respeito da ordem encontra-se disponibilizada em formato PDF no sítio Ephemeroptera Galactica (http://www.famu.org/mayfly/).

Todo o material encontra-se depositado na Coleção do Laboratório de Diversidade de Insetos Aquáticos da Universidade Federal do Espírito Santo, em São Mateus (ES). As únicas exceções constituem os materiais-tipo dos novos táxons. Maiores informações acerca de 


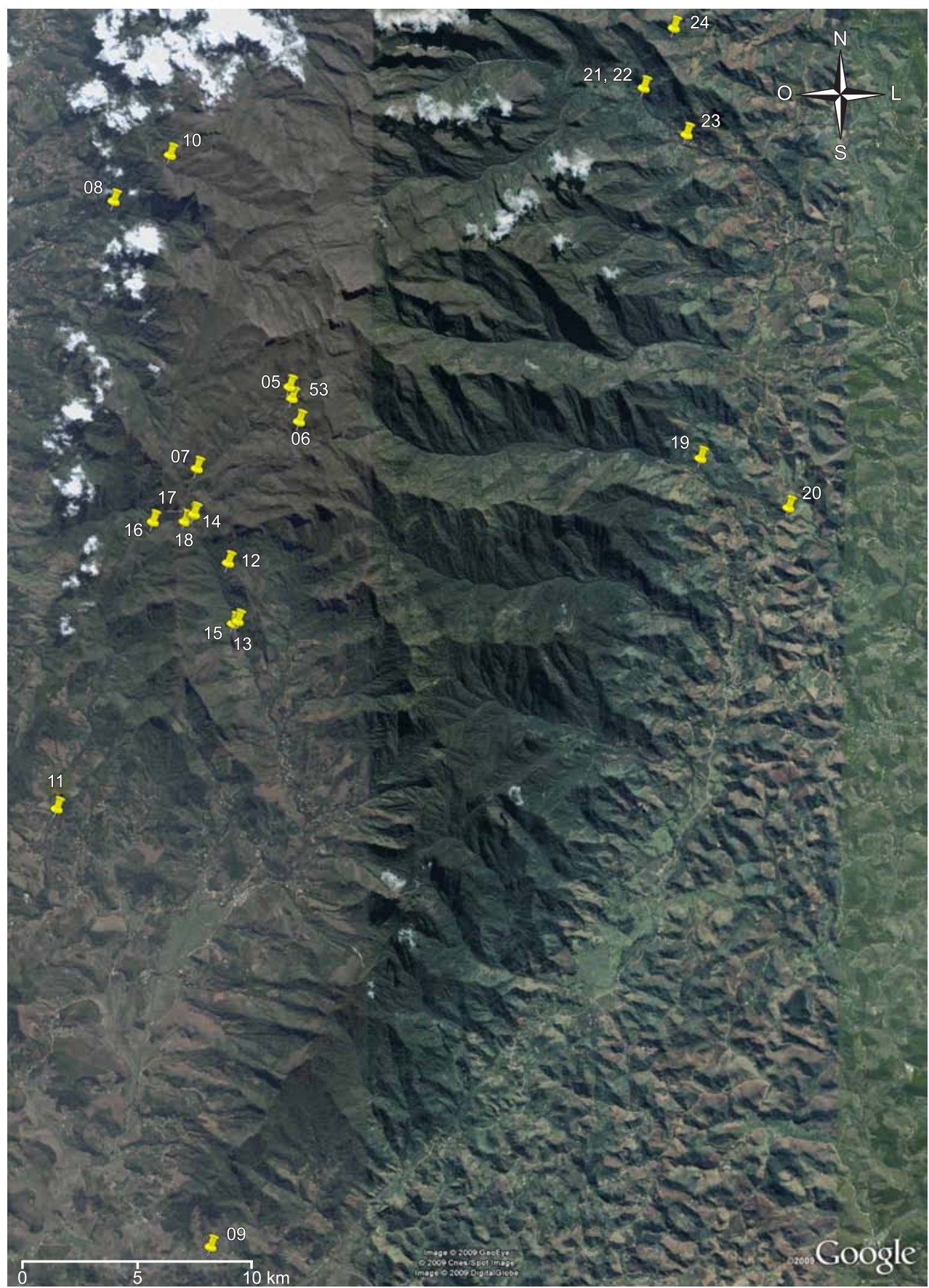

Figura 2. Imagem de satélite do Parque Nacional do Caparaó, ES, com distribuição dos pontos de coleta realizadas (para maiores detalhes vide Tabela 1) (fonte: Google Maps).

Figure 2. Satellite image of Parque Nacional do Caparaó, ES, showing the distribution of collection stations (to see more details, check Table 1) (source: Google Maps).

sua deposição podem ser encontradas nos artigos pertinentes às suas descrições.

\section{Material examinado e distribuição geográfica}

Para elaboração da distribuição geográfica das espécies foram consultados Domínguez et al. (2006) e Salles (2009). No item material examinado, PT se refere ao ponto de coleta (Tabela 1), entre parênteses apresentamos o número de indivíduos seguido do estágio examinado, $\mathrm{N}$ para ninfas e A para adultos e, finalmente, a data de coleta.

\section{Resultados}

Com base na literatura e nos táxons amostrados, apresentamos a seguir uma lista contendo todas as espécies ou morfo-espécies de Ephemeroptera registradas para o Estado, os quais totalizaram 76 espécies, 41 gêneros e nove famílias (Apêndice 1). Para cada uma das espécies listadas, dados acerca da sua distribuição geográfica são apresentados juntamente com a listagem dos pontos no Espírito Santo onde foram coletadas (maiores detalhes sobre os pontos de coleta são apresentados na Tabela 1). Comentários, quando pertinentes, também são abordados. Com o intuito de atualizar a lista de Ephemeroptera 


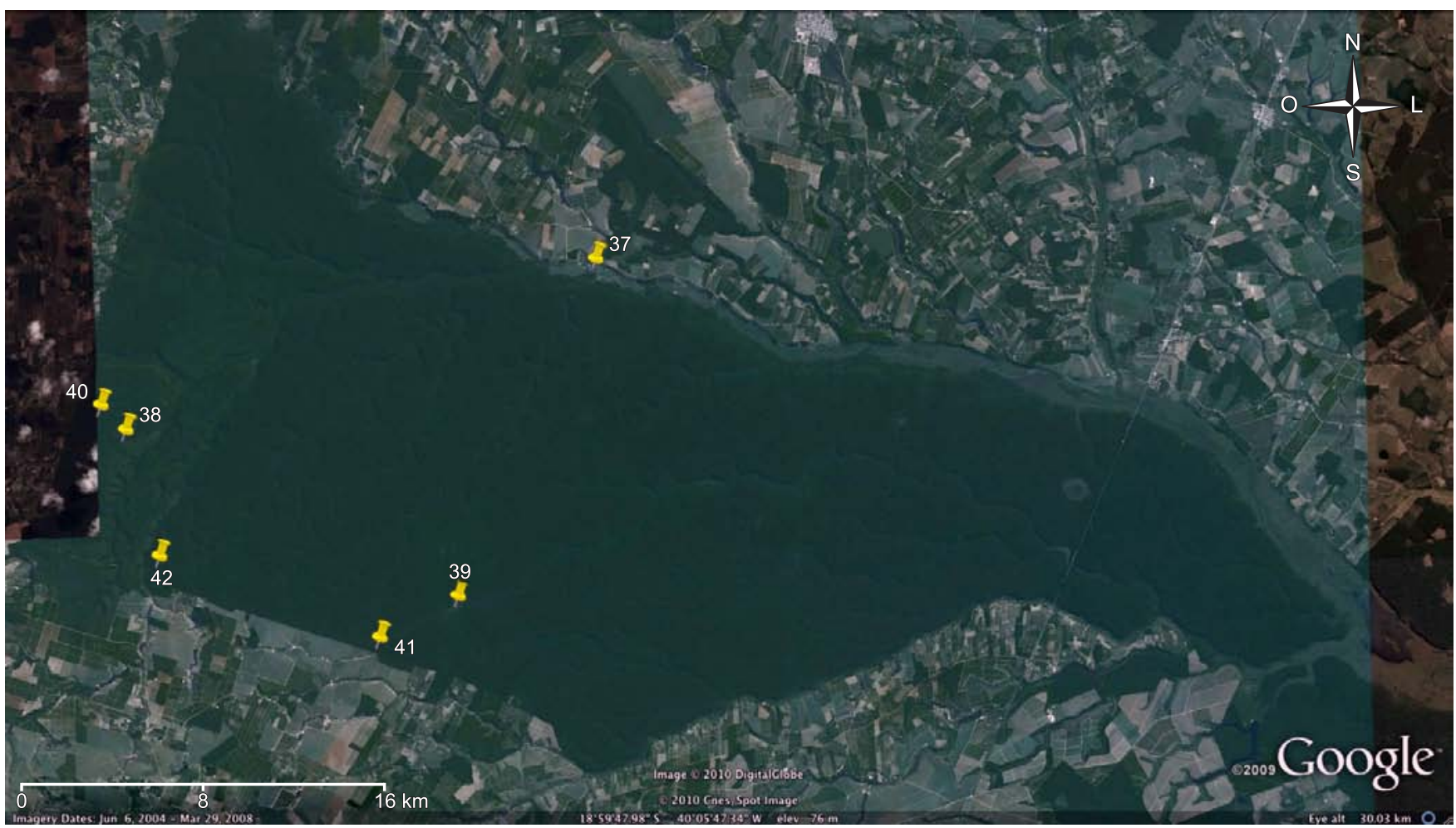

Figura 3. Imagem de satélite da Reserva Biológica de Sooretama, ES, com distribuição dos pontos de coleta realizadas no presente trabalho (para maiores detalhes vide Tabela 1) (fonte: Google Maps).

Figure 3. Satellite image of Reserva Biológica de Sooretama, ES, showing the distribution of collection stations (to see more details, check Table 1) (source: Google Maps).

do Espírito Santo, a mesma está sendo disponibilizada na internet a partir do sítio Diversidade de Insetos Aquáticos (http://sites.google. com/site/insetosaquaticos/).

\section{Discussão}

Apenas 12 espécies, distribuídas em 10 gêneros e cinco famílias encontravam-se registradas para o Espírito Santo até o presente trabalho. Neste, apresentamos novos registros de 64 espécies, 31 gêneros e quatro famílias. Seguindo os padrões de diversidade de Ephemeroptera para América do Sul e Brasil (Salles et al. 2004), as famílias que apresentaram a maior proporção de registros foram Baetidae (Figuras 8-9) 38\% das espécies $(n=29)$ e $34 \%$ dos gêneros (14), Leptophlebiidae (Figuras 4-6) 29\% (22) e 39\% (16), e Leptohyphidae com $21 \%$ (16) e $12 \%$ (5), respectivamente. Caenidae, Euthyplociidae e Polymitarcyidae (Figura 7) foram representadas por um gênero e duas espécies cada, enquanto Oligoneuriidae, Ephemeridae (Figuras 10-11) e Melanemerellidae por um gênero e uma espécie. Vale ressaltar, entretanto, que Melanemerellidae é monotípica (Molineri \& Domínguez 2003), Ephemeridae possui apenas uma espécie registrada para o país (das três que ocorrem na América do Sul) e Euthyplociidae somente quatro (Domínguez et al. 2006). O número de espécies de Caenidae e Polymitarcyidae e de espécies e gêneros de Oligoneuriidae, contudo, deve ser maior. O hábito críptico das ninfas, muitas das quais vivem enterradas ou semi-enterradas (Domínguez et al. 2006, Salles 2006, Salles et al. 2009), a dificuldade em se identificar as espécies de alguns de seus gêneros com base em imaturos (principalmente Polymitarcyidae e Oligoneuriidae), aliada à

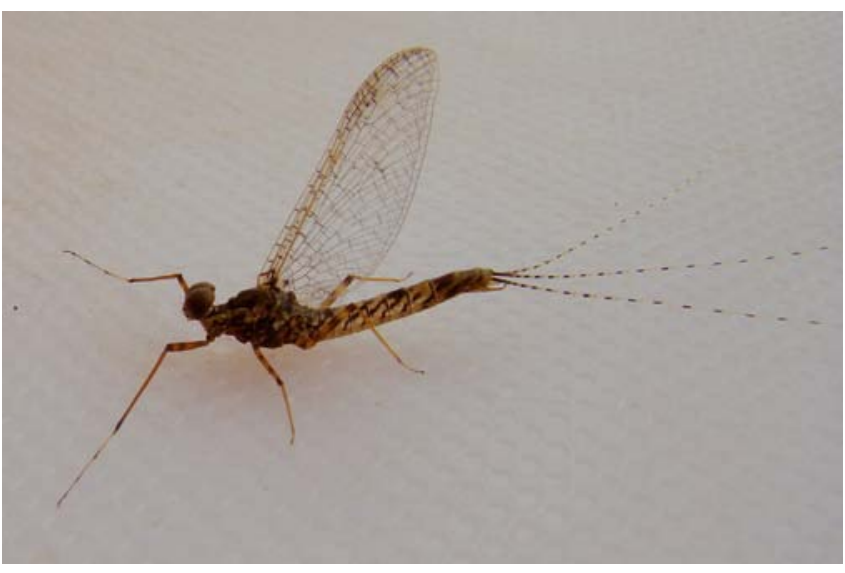

Figura 4. Imago macho de Massartella brieni (Lestage, 1924) (Leptophlebiidae), município de Santa Teresa, ES.

Figure 4. Male imago of Massartella brieni (Lestage, 1924) (Leptophlebiidae), municipality of Santa Teresa, ES.

diversidade desses grupos no Brasil e América do Sul (Salles 2009), corroboram esta hipótese.

De todas as espécies coletadas, cinco não puderam ser identificadas: uma de Baetidae (Callibaetis), três de Leptophlebiidae (Thraulodes) e uma de Oligoneuriidae (Lachlania). Em todos esses gêneros a taxonomia é baseada principalmente no estágio adulto, poucas ninfas foram descritas até o momento e todos apresentam um número alto de espécies descritas, especialmente Callibaetis e Thraulodes (Domínguez et al. 2006). Portanto, a chance de serem 


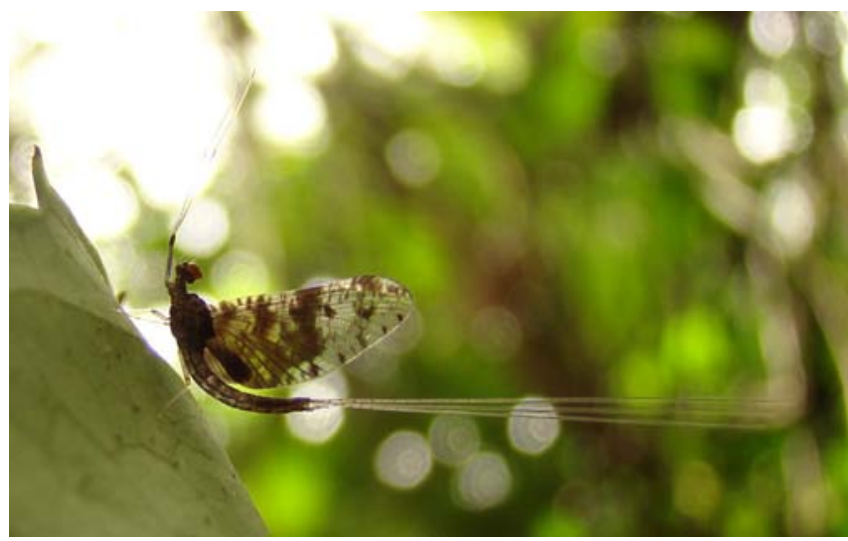

Figura 5. Imago macho de uma nova espécie de Miroculis Edmunds (Leptophlebiidae) encontrada no Parque Nacional do Caparaó, ES.

Figure 5. Male imago of a new species of Miroculis Edmunds (Leptophlebiidae) found at Parque Nacional do Caparaó, ES.

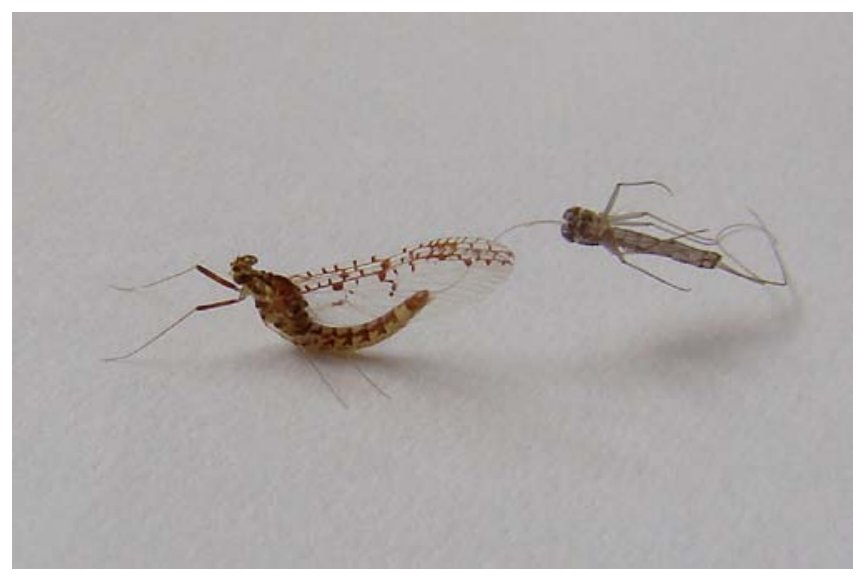

Figura 6. Imago macho de Fittkaulus cururuensis Savage, 1986 (Leptophlebiidae) com exúvia subimaginal, Reserva Biológica de Sooretama, município de Sooretama, ES.

Figure 6. Male imago of Fittkaulus cururuensis Savage, 1986 (Leptophlebiidae) with subimaginal exuvia, Reserva Biológica de Sooretama, municipality of Sooretama, ES.

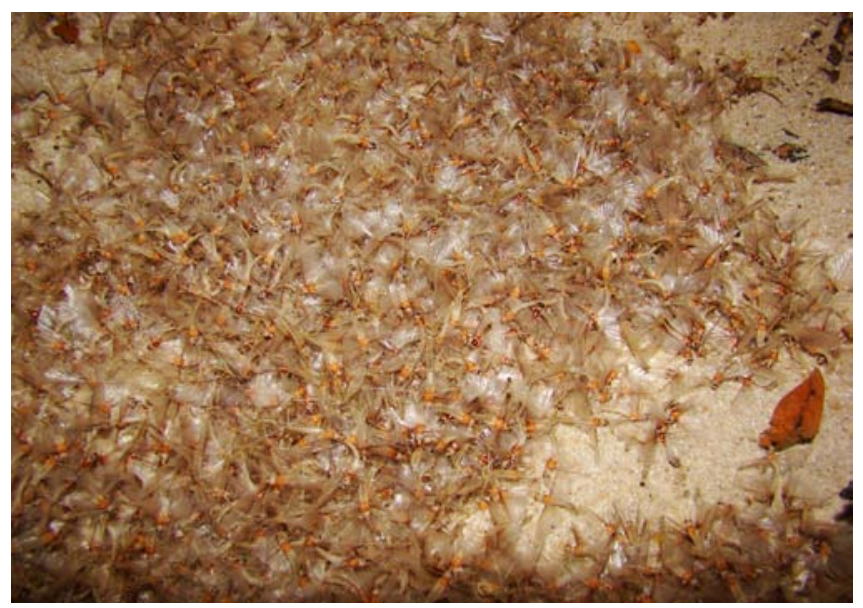

Figura 7. Imagos macho de Campsurus latipennis (Walker, 1853) durante revoada na Lagoa Nova, Linhares, ES.

Figure 7. Male imagos of Campsurus latipennis (Walker, 1853) during swarm at Lagoa Nova, Linhares, ES.

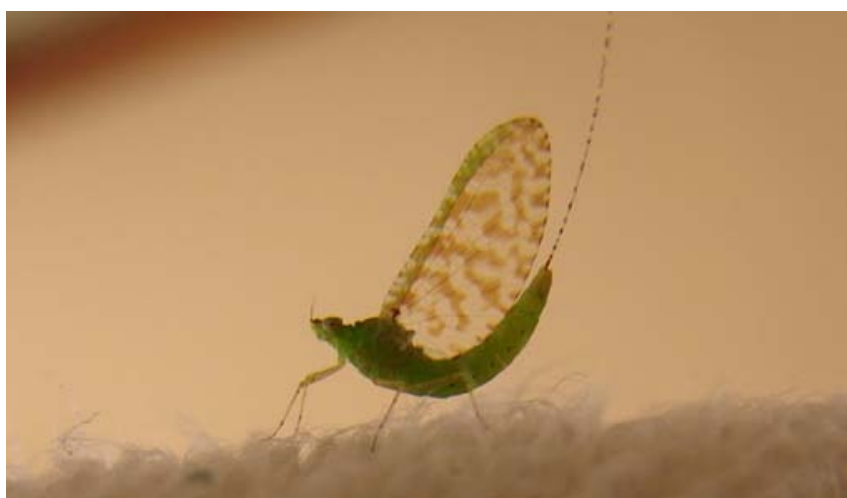

Figura 8. Imago fêmea de Callibaetis guttatus Navás, 1925 (Baetidae); espécie frequentemente encontrada no município de São Mateus, ES.

Figura 8. Female imago of Callibaetis guttatus Navás, 1925 (Baetidae); a species of mayfly often found at São Mateus city, ES.

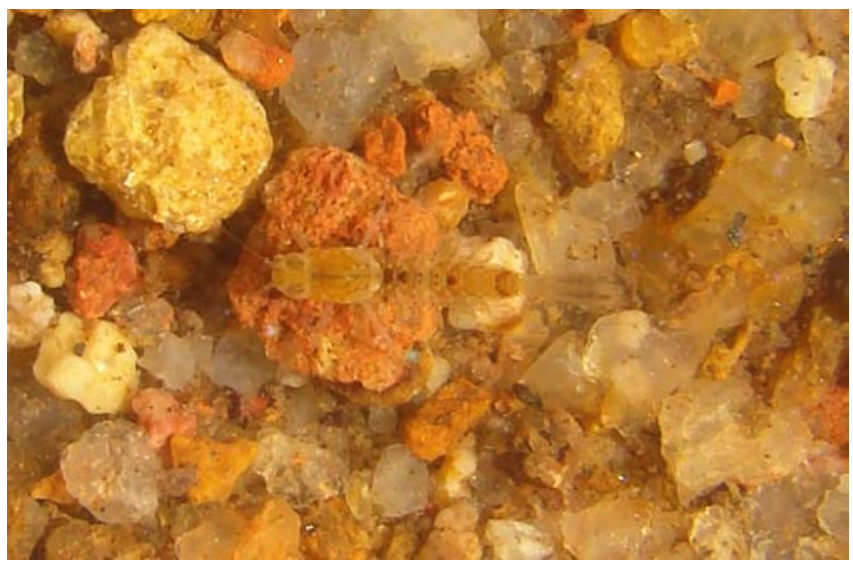

Figura 9. Ninfa de Rivudiva minantenna Lugo-Ortiz \& McCafferty, 1998 (Baetidae) camuflada sobre cascalho e areia, município de Santa Teresa, ES.

Figure 9. Nymph of Rivudiva minantenna Lugo-Ortiz \& McCafferty, 1998 (Baetidae) camouflaged over gravel and sand, Santa Teresa city, ES.

encontradas ninfas desconhecidas de espécies descritas com base apenas em adultos é alta. De qualquer forma, não deve ser descartada a possibilidade de que essas espécies sejam novas.

Ao longo desses dois anos de coleta, um total de 17 novos táxons confirmados foi encontrado: 11 espécies de Baetidae, quatro espécies de Leptophlebiidae, e duas de Leptohyphidae. Excluindo-se as espécies novas e ainda não descritas (17), somadas àquelas não identificadas (cinco), 54 espécies nominais, distribuídas em 41 gêneros e nove famílias encontram-se registradas para o Espírito Santo. A título de comparação, os estados brasileiros até o presente trabalho com o maior número de registros de Ephemeroptera eram Rio de Janeiro (30 gêneros e 51 espécies), Amazonas (31gêneros e 43 espécies), São Paulo (27 gêneros e 35 espécies) e Santa Catarina (20 gêneros e 37 espécies) (Salles 2009). Ressaltamos, ainda, que nesses estados pesquisas com insetos aquáticos são realizadas há relativamente um longo tempo (Rio de Janeiro, São Paulo e Amazonas) e/ou associadas a pesquisadores que realizaram importantes coletas nas regiões (Dr. E. Fittkau, Amazonas e F. Plaumann, Santa Catarina). Outro fator que demonstra a relevância dos resultados obtidos pode ser evidenciado quando comparamos os valores encontrados no estado aos do Brasil. Para o país são conhecidas 203 espécies, 67 gêneros 
e 10 famílias (Salles 2009), de maneira que estão representados no estado mais de $1 / 4$ das espécies brasileiras, cerca de $2 / 3$ dos gêneros e quase todas as famílias.

Das 12 espécies que estavam registradas para o Espírito Santo anteriormente a esse trabalho, Leptohyphes plaumanni, L. cornutus, Tricorythopsis araponga, T. pseudogibbus (Leptohyphidae), Melanemerella brasiliana (Melanemerellidae) e Campylocia anceps (Euthyplociidae) não foram encontradas, enquanto Americabaetis longetron, Paracloedes eurybranchus, Waltzoyphius fasciatus (Baetidae) e Campsurus truncatus (Polymitarcyidae) foram amostradas. Os registros prévios de Cloeodes irvingi e Zelusia principalis (Baetidae) (Lugo-Ortiz et al., 2002) devem ser desconsiderados, uma vez constatado que os indivíduos apontados como pertencentes a essas espécies tratam-se, de fato, de espécies desconhecidas para a ciência.

Para a maior parte das espécies os registros representam pequenas ampliações dos seus padrões de distribuição, em geral a norte ou a leste. No entanto, algumas espécies apresentaram ampliações significativas no que diz respeito às suas distribuições prévias. É o caso de Cloeodes opacus (Baetidae), previamente registrada somente para Argentina (Nieto \& Richard 2008), e de uma espécie de Caenidae (Caenis fittkaui), cinco espécies de Leptophlebiidae (Fittkaulus cururuensis, Hydrosmilodon gilliesae, Miroculis fittkaui, Simothraulopsis (M.) plesius, S. (S.) demerara) e uma de Polymitarcyidae (Campsurus latipennis), todas, até então, conhecidas apenas na Região Amazônica.

A despeito do seu pequeno tamanho, pois a área do estado representa apenas $0,53 \%$ do território nacional, uma grande diversidade de Ephemeroptera foi encontrada. De um dos estados menos conhecidos do Brasil, o Espírito Santo torna-se, a partir dos dados apresentados, o estado brasileiro com o maior número de gêneros e espécies da ordem. Com relação às famílias, iguala-se ao Amazonas e ao Rio de Janeiro, uma vez que das 10 famílias registradas para o país, apenas Coryphoridae, restrita até o momento à Bacia Amazônica, não foi registrada.

Dentre os motivos para tal diversidade podemos sugerir algumas hipóteses, bem como o efeito conjunto das mesmas. Primeiramente, a heterogeneidade dos locais amostrados, que variaram desde áreas ao nível do mar até 2.250 metros de altitude; diferentes fitofisionomias, tais como restingas, florestas ombrófilas densas de terras baixas (Matas de Tabuleiro) e altimontana, campos de altitude, etc; e ambientes aquáticos com diferentes características, como lênticos (desde brejos e pequenas poças a grandes lagoas) e lóticos (variando de nascentes a cachoeiras, ou córregos e rios com fundo arenoso ou pedregoso). Aliada a isso, uma outra hipótese, mas que aguarda estudos mais focados nesse sentido, está relacionada à presença no estado de dois componentes faunísticos distintos. Um, típico do sudeste da América do Sul, frequentemente encontrado nos estados de Santa Catarina, Rio de Janeiro, São Paulo e Minas Gerais, como os gêneros Thraulodes, Hylister, Needhamella, Tupiara, Leptohyphodes, e outro componente até então mais restrito à fauna amazônica e não encontrado nos estados supracitados, como Adebrotus, Terpides, Fittkaulus, Simothraulopsis, Hydrosmilodon e uma espécie de Miroculis. Por fim, a diversidade encontrada no presente trabalho reflete o próprio esforço amostral e de identificação dos táxons como exemplificado a seguir. Apesar de poucas coletas terem sido realizadas, uma equipe de ao menos quatro pesquisadores esteve envolvida exclusivamente na coleta de Ephemeroptera em cada uma das campanhas. A identificação dos táxons, além de ter sido realizada por ao menos sete especialistas na ordem, muitas vezes só foi possível em função do esforço empreendido na criação das ninfas e consequente associação com adultos, imprescindível para a identificação de alguns táxons, especialmente Leptophlebiidae. Aliado a esses fatores, ressaltamos ainda a existência de chaves de identificação atualizadas e próprias para a região (e.g. Dominguez et al. 2006, Salles 2006), ferramentas inexistentes há poucos anos atrás, e a facilidade em se obter literatura especializada, uma vez que praticamente todos os artigos que abordam taxonomia de Ephemeroptera na América do Sul encontram-se disponíveis na internet.

\section{Agradecimentos}

Gostaríamos de agradecer a Angela Brunner da Rocha, por apoio logístico e por nos permitir trabalhar em sua propriedade, e Pedro Brito pela ajuda durante as coletas; às equipes da Reserva Biológica de Sooretama, Parque Nacional do Caparaó, Reserva Biológica do Córrego do Veado e Reserva Biológica Augusto Ruschi; ICMBio (Instituto Chico Mendes de Conservação da Biodiversidade) e IBAMA (Instituto Brasileiro do Meio Ambiente e dos Recursos Naturais Renováveis) pelas permissões de coleta (números 12777-1, 16719-1 e 11239-1); Fundação de Apoio à Ciência e Tecnologia do Espírito Santo (FAPES), pelo apoio financeiro ao projeto "Diversidade de Ephemeroptera em duas Unidades de Conservação do Espírito Santo" (processo número 36327263/2007); CNPq (Conselho Nacional para o Desenvolvimento de Pesquisa Científica e Tecnológica) e UFES (Universidade Federal do Espírito Santo), programa PIBIC/ PIVIC 2008/2009, 2009/2010, pela concessão de bolsas de iniciação científica; e a Elaine Della Giustina Soares (CEUNES/UFES) pela confecção do mapa do Espírito Santo e sugestões ao artigo.

\section{Referências}

BOLDRINI, R. \& SALLES, F.F. 2009. A new species of two-tailed (Insecta: Emphemeroptera: Baetidae) from the State Espírito Santo. Rev. Mus. Biol. Mello Leitão 25:5-12.

BOLDRINI, R., SALLES, F.F. \& CABETTE, H.R.S. 2009. Contribution to the taxonomy of the Terpides lineage (Ephemeroptera: Leptophlebiidae). Ann. Limnol. Int. J. Lim. (no prelo)

BOLDRINI, R., SALLES, F.F. \& PES, A.M. 2010. Imagos of Camelobaetidius francischettii Salles, Andrade \& Da-Silva (Ephemeroptera: Baetidae). Zootaxa (no prelo)

COSTA, J.M. \& OLDRINI, B.B. 2005. Diversidade e distribuição dos Odonata (Insecta) no Estado do Espírito Santo, Brasil. Pub. Avul. Mus. Nac. 107:1-15.

CRUZ, P.V., SALLES, F.F. \& HAMADA, N. 2009. Two new species of Callibaetis Eaton (Ephemeroptera: Baetidae) from Southeastern Brazil. Zootaxa 2261:23-38.

DE SOUZA, M.R., SALLES, F.F. \& NESSIMIAN, J.L. 2010. Three new species of Baetodes Nedham \& Murphy (Ephemeroptera: Beatidae) from Southern Brazil. Aquat. Insects (no prelo)

DIAS, L.G., MOLINERI, C. \& FERREIRA, P.S.F. 2007. Ephemerelloidea (Insecta: Ephemeroptera) do Brasil. Pap. Avul. Zool. 47(19):213-244.

DOMÍNGUEZ, E., MOLINERI, C., PESCADOR, M.L., HUBBARD, M.D. \& NIETO, C. 2006. Ephemeroptera of South America. In Aquatic Biodiversity of Latin America (J. Adis, J.R. Arias, G. Rueda-Delgado \& K.M. Wantzen, eds.). Pensoft, Moscow-Sofia, p. 1-646. (v. 2)

INSTITUTO BRASILEIRO DO MEIO AMBIENTE E DOS RECURSOS NATURAIS RENOVÁVEIS - IBAMA. 2007. Parque Nacional do Caparaó. Brasília. http://www.ibama.gov.br/revista/caparao/texto_ caparao.htm (último acesso 26/11/2009).

INSTITUTO DE PERMACULTURA E ECOVILAS DA MATA ATLÂNTICA - IPEMA. 2007. Conservação da Mata Atlântica no Estado do Espírito Santo: cobertura florestal e unidades de conservação. 1 ed. Vitória, p. 1-152.

KIMMINS, D.E. 1960. The Ephemeroptera types of species described by A. E. Eaton, R. McLachlan and F. Walker, with particular reference to those in the British Museum (Natural History). Bull. Br. Mus. Nat. Hist. Entomol. 9:269-318. 
LUGO-ORTIZ, C.R., SALLES, F.F. \& FURIERI, K.S. 2002. First records of small minnow mayflies (Ephemeroptera: Baetidae) from the state of Espírito Santo, southeastern Brazil. Lundiana 3(1):79-80.

MINISTÉRIO DO MEIO AMBIENTE - MMA. 2008. Livro vermelho da fauna brasileira ameaçada de extinção. In (A.B.M. Machado, G.M. Drummond \& A.P. Paglia, orgs.). Brasília, 511 p.

MOLINERI, C. \& DOMÍNGUEZ, E. 2003. Nymph and egg of Melanemerella brasiliana (Ephemeroptera: Ephemerelloidea: Melanemerellidae), with comments on its systematic position and the higher classification of Ephemerelloidea. J. N. Am. Benthol. Soc. 22(2):263-275.

MOLINERI, C. 2002. Cladistic analysis of the South American species of Tricorythodes (Ephemeroptera: Leptohyphidae) with the description of new species and stages. Aquat. Insects 24(4):273-308.

NIETO, C. \& RICHARD, B. 2008. The genus Cloeodes (Ephemeroptera: Baetidae) in Argentina with new generic synonymy and new species. Zootaxa 1727:1-21.

SALLES, F.F. \& BOLDRINI, R. 2008. Male imago description of Americabaetis longetron Lugo-Ortiz \& McCafferty (Ephemeroptera: Baetidae), and first key to adults of the genus. Neotrop. Entomol. 37(5):564-566.

SALLES, F.F. \& NASCIMENTO, J.M.C. 2009. The Genus Rivudiva LugoOrtiz and McCafferty (Ephemeroptera: Baetidae): first generic description of adults, new combinations, and notes on the nymphs. Ann. Limnol. Int. J. Lim. (no prelo)

SALLES, F.F. 2006. A ordem Ephemeroptera (Insecta) no Brasil: diversidade e taxonomia. Tese de Doutorado, Universidade Federal de Viçosa, Minas Gerais.

SALLES, F.F. 2009. Lista das espécies de Ephemeroptera registradas para o Brasil. http://ephemeroptera.br.googlepages.com/home (último acesso em 20/09/2009).

SALLES, F.F., SILVA, E.R., HUBBARD, M.D. \& SERRÃO, J.E. 2004. As espécies de Ephemeroptera (Insecta) registradas para o Brasil. Biota Neotrop. 4(2): http://www.biotaneotropica.org.br/v4n2/pt/abstract?inv entory+BN04004022004

SALLES, F.F., FRANCISCHETTI, C.N. \& SOARES, E.D.G. 2009. The presence of Homoeoneuria s.s. (Ephemeroptera: Oligoneuriidae) in South America with the description of a new species. Zootaxa 2146:53-60.

ULMER, G. 1920. Neue Ephemeropteren. Arch. Naturg. 85(11):1-80.

ULMER, G. 1942. Alte und neue Eintagsfliegen (Ephemeropteren) aus Südund Mittelamerika. Stett. Ent. Zeitung 103:98-128.

Recebido em 03/12/09

Versão reformulada recebida em 12/03/10

Publicado em 18/03/10 
Apêndice 1. Lista das espécies de Ephemeroptera registradas para o Espírito Santo, acompanhada de distribuição geográfica, comentários e material examinado. Appendix 1. List of the species of Ephemeroptera reported from Espírito Santo, followed by geographic distribution, comments and material examined.

\section{BAETIDAE}

Adebrotus sp.n.

Distribuição geográfica: Brasil: Espírito Santo (Sooretama). Material examinado: PT 42: (1N) 02.vi.2009; PT 42: (1N) 02.vii.2009.

Americabaetis alphus Lugo-Ortiz \& McCafferty, 1996

Distribuição geográfica: Argentina, Bolívia, Chile, Paraguai, Brasil: Mato Grosso, Paraná, Santa Catarina, Rio Grande do Sul, Minas Gerais, Rio de Janeiro, São Paulo, Espírito Santo (Alto Caparaó, Ibitirama, Santa Teresa).

Comentários: Primeiro registro para o Espírito Santo.

Material examinado: PT 06: (34N) 25.iii.2009; PT 19: (2N)

20.iv.2008; PT 45: (1N) 19.i.2008; PT 45: (1N) 20.ii.2009 PT 47: (2N) 20.i.2008.

Americabaetis labiosus Lugo-Ortiz \& McCafferty, 1996

Distribuição geográfica: Paraguai, Uruguai, Brasil: Paraná, Santa Catarina, Rio Grande do Sul, Rio de Janeiro, Espírito Santo (Alfredo Chaves, Santa Teresa, Sooretama).

Comentários: Primeiro registro para o Espírito Santo.

Material examinado: PT 04: (6N) 23.viii.2008; PT 42: (1N)

29.iv.2009; PT 45: (1N) 20.ii.2009; PT 47: (5N) 20.i.2008.

Americabaetis longetron Lugo-Ortiz \& McCafferty, 1996

Distribuição geográfica: Paraguai, Uruguai, Brasil: Paraná, Santa Catarina, Rio de Janeiro, Minas Gerais, Espírito Santo (Alfredo Chaves, Ibitirama)

Comentários: Espécie previamente registrada para o estado

(Lugo-Ortiz et al. 2002, Salles \& Boldrini 2008).

Material examinado: PT 04: (2N) 23.viii.2008; PT 19: (11N) 21.iv.2008.

Americabaetis titthion Lugo-Ortiz \& McCafferty, 1996

Distribuição geográfica: Brasil: Paraná, Santa Catarina, Rio

Grande do Sul, Rio de Janeiro, Espírito Santo (Alto Caparaó)

Comentários: Primeiro registro para o Espírito Santo.

Material examinado: PT 06: (10N) 23.iv.2008; PT 08: (53N) 23.iv.2009.

Apobaetis fiuzai Salles \& Lugo-Ortiz, 2002

Distribuição geográfica: Argentina, Brasil: Mato Grosso, Minas Gerais, Rio de Janeiro, São Paulo, Espírito Santo (Alto Caparaó, Santa Teresa)

Comentários: Primeiro registro para o Espírito Santo.

Material examinado: PT 06: (10N) 23.iv.2008; PT 45: (1N) 20.i.2008; PT 45: (1N) 25.x.2008; PT 45: (4N) 26.x.2008.

Aturbina georgei Lugo-Ortiz \& McCafferty, 1996

Distribuição geográfica: Colômbia, Guiana Francesa, Paraguai, Brasil: Mato Grosso, Amazonas, Pará, São Paulo, Minas Gerais, Rio de Janeiro, Espírito Santo (Fundão, Ibitirama, Santa Teresa, São Mateus, Sooretama).

Comentários: Primeiro registro para o Espírito Santo.

Material examinado: PT 19: (1N) 20.iv.2008; P28: (10N, 20A) 12.ix.2009; PT 32: (2N) 30.i.2008; PT 39: (1N) 14.vii.2008; PT 42: (1N) 02.vi.2009; PT 45: (12N) 20.i.2008; PT 47: (1N) 20.i.2008; PT 50: (2N) 23.vii.2008.
Baetodes sp.n. 1

Distribuição geográfica: Brasil: Espírito Santo (Alfredo Chaves, Santa Teresa).

Material examinado: PT 04: (1N) 13.vii.2007; PT 45: (2N) 18.i.2008; PT 45: (17N) 19.i.2008.

Baetodes sp.n. 2

Distribuição geográfica: Brasil: Espírito Santo (Alfredo Chaves, Santa Teresa).

Material examinado: PT 04: (3N) 24.xii.2007; PT 45: (10N) 18.i.2008; PT 45: (1N) 19.i.2008.

Baetodes sp.n. 3

Distribuição geográfica: BRASIL: Espírito Santo (Ibitirama). Material examinado: PT 22: (8N) 19.i.2008.

Callibaetis capixaba Cruz, Salles \& Hamada, 2009

Distribuição geográfica: Brasil: Espírito Santo (Santa Teresa).

Comentários: Espécie recentemente descrita (Cruz et al. 2009).

Material examinado: PT 45: (5N) 20.i.2008; PT 45: (1N) 25.x.2008; PT 45: (38N) 26.x.2008; PT 48: (5N) 20.ii.2009.

Callibaetis guttatus Navás, 1915

Distribuição geográfica: Argentina, Brasil: Rio de Janeiro, Espírito Santo (São Mateus)

Comentários: Primeiro registro para o Espírito Santo.

Material examinado: PT 32: (17N) 12.ii.2007; PT 32: (2N)

22.iii.2007; PT 32: (66N) 27.x.2007; PT 32: (10N) 30.i.2008; PT 32: (1N) 25.ii.2008.

Callibaetis sp. 1

Distribuição geográfica: Brasil: Espírito Santo (Santa Teresa).

Comentários: Identificada com base em ninfas; sua associação aos adultos é imprescindível para que possa ser corretamente identificada.

Material examinado: PT 47: (2N) 20.i.2008.

Camelobaetidius anubis (Traver \& Edmunds, 1968)

Distribuição geográfica: Argentina, Brasit: Paraná, Santa Catarina, São Paulo, Minas Gerais, Rio de Janeiro, Espírito Santo (Alfredo Chaves, Santa Teresa)

Comentários: Espécie recentemente registrada para o estado com base em material oriundo de Alfredo Chaves (Boldrini \& Salles 2009).

Material examinado: PT 04: (5N) 13.vii.2007; PT 45: (10N) 20.ii.2009.

Camelobaetidius francischettii Salles, Andrade e Da-Silva, 2005

Distribuição geográfica: Brasil: Bahia, Espírito Santo (Alfredo Chaves)

Comentários: Espécie recentemente registrada para o Estado com base em material oriundo de Alfredo Chaves (Boldrini \& Salles 2009).

Material examinado: PT 04: (4N) 13.vii.2007.

Camelobaetidius rufiventris Boldrini \& Salles, 2009

Distribuição geográfica: Brasil: Espírito Santo (Alfredo Chaves). 
Comentários: Espécie recentemente descrita com base em material oriundo de Alfredo Chaves (Boldrini \& Salles 2009).

Material examinado: PT 04: (1N) 28.v.2007; PT 04: (9N) 23.ix.2007.

Camelobaetidius sp.n.

Distribuição geográfica: Brasil, Espírito Santo (Alfredo Chaves).

Material examinado: PT 04: (15N) 23.ix.2007.

Cloeodes hydation McCafferty \& Lugo-Ortiz, 1995

Distribuição geográfica: Brasil: Mato Grosso, Minas Gerais, Espírito Santo (Divisa ES/MG - Vale Encantado).

Comentários: Primeiro registro para o Espírito Santo.

Material examinado: PT 10: (4N) 24.iii.2009.

Cloeodes opacus Nieto, 2008

Distribuição geográfica: Argentina; Brasil, Espírito Santo (Alfredo Chaves, Ibitirama, Santa Teresa), Divisa Minas Gerais/ Espírito Santo (Espera Feliz).

Comentários: Primeiro registro para o Brasil.

Material examinado: PT 04: (2N) 27.v.2007; PT 04: (1N) 14.vii.2007; PT 04: (1A) 13.x.2007; PT 04: (1A) 04.xi.2007; PT 04: (7A) 23.xii.2007; PT 04: (9N) 24.xii.2007; PT 04: (2A) 03.ii.2008; PT 04: (9N) 23.viii.2008; PT 11: (7N) 27.iii.2009; PT 22: (1A) 20.iv.2008; PT 43: (1N) 20.i.2008; PT 45: (1N) 20.i.2008, (2N) 25.x.2008; PT 46: (1N) 26.x.2008; PT 47: (5N) 20.ii.2009.

Cloeodes sp.n. 1

Distribuição geográfica: Brasil: Espírito Santo (Alto Caparaó, Ibitirama, Santa Teresa), Divisa Minas Gerais/Espírito Santo (Espera Feliz).

Comentários: Espécie previamente registrada para o estado como C. irvingi Waltz \& McCafferty (Lugo-Ortiz et al. 2002). Após novo exame do material identificado por esses autores constatamos que trata-se de uma nova espécie.

Material examinado: PT 6: (2N) 23.iv.2009; PT 7: (29N) 23.iv.2008; PT 7: (27N) 25.iii.2009; PT 8: (11N) 23.iii.2009; PT 11: (3N) 27.iii.2009; PT 16: (21N) 22.iv.2008; PT 18: (25N) 22.iv.2008; PT 18: (8N) 25.iii.2009; PT 19: (15N) 21.iv.2008; PT 21: (2N) 20.iv.2008; PT 22: (10N, 1A) 20.iv.2008.

Cloeodes sp.n. 2

Distribuição geográfica: Brasil: Espírito Santo (Águia Branca, Alto Caparaó, Ibitirama, Iuna, Santa Teresa), Divisa Minas Gerais/Espírito Santo (Espera Feliz).

Material examinado: PT 1: (15N) 02.i.2009; PT 1: (26N) 23.ii.2009; PT 7: (7N) 23.iv.2008; PT 7: (5N) 25.iii.2009; PT 8: (2N) 23.iii.2009; PT 16: (2N) 22.iv.2008; PT 17: (1N) 23.iv.2008; PT 18: (7N) 25.iii.2009; PT 19: (1N) 21.iv.2008; PT 21: (3N) 20.iv.2008; PT 22: (2N) 21.iv.2008; PT 24: (17N) 21.iv.2008; PT 43: (1N) 20.i.2008; PT 43: (2N) 25.x.2008; PT 44: (1N) 16.xi.2007; PT 44: (1N) 19.i.2008; PT 44: (1N) 26.x.2008; PT 45: (2N) 25.x.2008; PT 45: (1N) 18.ii.2009; PT 46: (1N) 26.x.2008.

Cloeodes sp.n. 3

Distribuição geográfica: Brasil: Espírito Santo (Ibitirama, Iuna, Santa Teresa), Divisa Minas Gerais/Espírito Santo (Espera Feliz).

Material examinado: PT 11: (1N) 27.iii.2009; PT 19: (24N) 21.iv.2008; PT 20: (40N) 21.iv.2008; PT 21: (8N) 20.iv.2008; PT 22: (11N) 20.iv.2008; PT 23: (1N) 21.iv.2008; PT 24: (1N) 21.iv.2008; PT 43: (8N) 20.i.2008; PT 43: (1N) 25.x.2008; PT 43: (2N) 19.ii.2009; PT 44: (1N) 19.i.2008; PT 45: (1N) 19.i.2008; PT 45: (1N) 18.ii.2009; PT 46: (2N) 26.x.2008; PT 48: (5N) 20.ii.2009.
Cloeodes sp.n. 4

Distribuição geográfica: Brasil: Espírito Santo (Alto Caparaó,

Divisa ES/MG - Vale Encantado).

Material examinado: PT 6: (6N) 23.iv.2009; PT 10: (23N) 24.iii.2009.

Cryptonympha dasilvai Salles \& Francischetti, 2004

Distribuição geográfica: Brasil: Rio de Janeiro, São Paulo,

Espírito Santo (Alfredo Chaves, Santa Teresa)

Comentários: Primeiro registro para o Espírito Santo.

Material examinado: PT 02: (1N) 20.viii.2008; PT 03: (6N) 24.vii.2008; PT 04: (17N) 23.viii.2008; PT 45: (8N) 19.ii.2009; PT 45: (10N) 20.ii.2009; PT 47: (15N) 20.ii.2009; PT 48: (10N) 20.ii.2009.

Paracloeodes eurybranchus Lugo-Ortiz \& McCafferty, 1996

Distribuição geográfica: Argentina, Brasil: Rio Grande do Sul,

Rio de Janeiro, Minas Gerais, São Paulo, Espírito Santo (Alto Caparaó, Iúna, Santa Teresa, Sooretama), Divisa Minas Gerais/ Espírito Santo (Espera Feliz).

Comentários: Espécie previamente registrada para o Estado (Lugo-Ortiz et al. 2002).

Material examinado: PT 05: (10N) 26.iii.2009; PT 06: (2N) 23.iv.2008; PT 07: (2N) 25.iii.2009; PT 14: (3N) 25.iii.2009; PT 17: (16N) 25.iii.2009; PT 17: (14N) 22.iv.2009; PT 24: (49N) 21.iv.2008; P28: (8N) 12.ix.2009; PT 38: (7N) 29.iv.2009; PT 41: (1N) 15.ii.2009; PT 45: (1N) 18.i.2009; PT 45: (2N) 20.i.2008.

Paracloeodes sp.n.

Distribuição geográfica: BRASIL: Espírito Santo (Alfredo Chaves, Ibitirama Iúna, Santa Teresa, Sooretama).

Material examinado: PT 03: (5N) 24.vii.2008; PT 04: (2N) 23.viii.2008; PT 19: (21N) 21.iv.2008; PT 21: (1N) 20.iv.2008; PT 24: (49N) 21.iv.2008; PT 37: (4N) 14.vii.2008; PT 45: (1N) 21.i.2008.

Rivudiva minantenna Lugo-Ortiz \& McCafferty, 1998

Distribuição geográfica: Brasil: Rio Grande do Sul, Santa

Catarina, Rio de Janeiro, Espírito Santo (Santa Teresa)

Comentários: Espécie recentemente registrada para o estado com base em material oriundo de Santa Teresa (Salles \& Nascimento 2009).

Material examinado: PT 45: (8N) 24.x.2008; PT 45: (24N)

26.x.2008; PT 47: (14N) 20.ii.2009; PT 48: (2N) 20.ii.2009.

Tupiara ibirapitanga Salles, Lugo-Ortiz, Da-Silva \& Francischetti, 2003

Distribuição geográfica: BRASIL: Amazonas, Minas Gerais ,

Rio de Janeiro, Espírito Santo (Alfredo Chaves, Ibitirama)

Comentários: Primeiro registro para o Espírito Santo.

Material examinado: PT 06: (9N) 23.iv.2008; PT 19: (12N)

21.iv.2008; PT 22: (24N) 20.iv.2008.

Waltzoyphius fasciatus McCafferty \& Lugo-Ortiz, 1995

Distribuição geográfica: Colômbia, Paraguai, Brasil: Minas Gerais, Rio de Janeiro, Espírito Santo (Alfredo Chaves, Conceição da Barra, São Mateus, Sooretama).

Comentários: Espécie previamente registrada para o estado (Lugo-Ortiz et al. 2002).

Material examinado: PT 02: (6N) 23.viii.2008; PT 27: (1N) 15.iv.2008; PT 32: (5N) 30.i.2008; PT 37: (1N) 30.iv.2008; PT 39: (2N) 14.vii.2008; PT 40: (46N) 15.vi.2008; PT 41: (1N) 14.vii.2008; PT 41: (1N) 15.vii.2008; PT 45: (1N) 18.i.2008 PT 45: (1N) 26.x.2008. 


\section{Zelusia sp.n.}

Distribuição geográfica: BRASIL: Espírito Santo (Alfredo Chaves, Sooretama, Santa Teresa, Ibitirama, Iúna), Divisa Espírito Santo/Minas Gerais (Espera Feliz).

Comentários: Espécie previamente registrada para o estado (Lugo-Ortiz et al. 2002) como Z. principalis Lugo-Ortiz \& McCafferty. Contudo, após análise de uma série maior de ninfas, incluindo aquelas identificadas por Lugo-Ortiz et al. (2002), e de comparação com material proveniente da localidade-tipo de Z. principalis, única espécie descrita do gênero, concluímos que se trata de uma nova espécie.

Material examinado: PT 03: (9N) 24.vii.2001; PT 04: (N) 23.viii.2008; PT 05: (1N) 26.iii.2009; ; PT 05: (1N) 26.iii.2009; ; PT 05: (8N) 26.iii.2009; ; PT 05: (1N) 26.iii.2009; PT 07: (1N) 25.iii.2009; PT 07: (1N) 25.iii.2009; PT 13: (1N) 25.iii.2009; PT19 : (N) 24.iv.2008; PT 21: (1N) 24.iv.2008; PT 21: (4N) 24.iv.2008; PT 21: (1N) 24.iv.2008; PT 24: (1N) 21.iv.2008; PT 24: (4N) 21.iv.2008; PT 39: (1N) 18.xii.2009; PT 40: (3N ) 15.vii.2008; PT 41: (1N) 14.vii.2008; PT 41: (2N) 30.iv.2209; PT 43: (1N) 20.i.2008; PT 43: (2N) 20.i.2008; ; PT 43: (4N) 20.i.2008; PT 44: (4N) 16.xi.2007; PT 44: (1N) 19.i.2008; PT 44: (2N) 26.x.2008; PT 44: (1N) 19.ii.2009; PT 45: (2N) 20.i.2008; PT 45: (1N) 18.i.2008; PT 45: (2N) 18.i.2009; PT 45: (1N) 18.ii.2009; PT45: (1N) 26.x.2008; PT45: (2N) 26.x.2008; PT 45: (1N) 20.ii.2009; PT 45: (5N) 20.ii.2009; PT 50: (1N) 23.ii.2007.

\section{CAENIDAE}

Caenis cuniana Froehlich, 1969

Distribuição geográfica: Brasil: Rio de Janeiro, São Paulo, Espírito Santo (Conceição da Barra, São Mateus, Sooretama).

Comentários: Primeiro registro para o Espírito Santo.

Material examinado: PT 25: (1N) 15.iv.2008; PT 30: (33N) 02.x.2007; PT 32: (1N) 22.vi.2007; PT 33: (15N) 10.xi.2007; PT 34: (1N) 20.xi.2007; PT 38: (1N) 29.iv.2009; PT 41: (4N) PT 41: 14.vii.2008; PT 41: (2N) 15.vii.2008; PT 41: (15N) 30.iv.2009.

Caenis fittkaui Malzacher, 1986

Distribuição geográfica: Brasil: Pará, Espírito Santo (Águia Branca, Pinheiros, São Mateus, Sooretama)

Comentários: Primeiro registro para a Região Sudeste.

Material examinado: PT 01: (1N) 02.i.2009; PT 32: (1N) 10.iii.2007; PT 32: (3N) 22.vi.2007; PT 32: (8N) 30.i.2008; PT 33: (5N) 10.xi.2007; PT 34: (1N) 20.xi.2007; PT 36: (13N) 13.iii.2008; PT 37: (1N) 14.vii.2008; PT 38: (7N) 29.iv.2009; PT 39: (1N) 18.xii.2008; PT 41: (1N) 14.vii.2008; PT 41: (2N) 15.vii.2008; PT 41: (7N) 30.iv.2009; PT 51: (3N) 03.ix.2009.

\section{EPHEMERIDAE}

Hexagenia (Pseudoeatonica) albivitta (Walker, 1853)

Distribuição geográfica: América Central, Argentina, Colômbia, Guiana, Paraguai, Uruguai, Brasil: Paraná, São Paulo, Pará, Espírito Santo (Linhares).

Comentários: Espécie aparentemente comum na Lagoa Juparanã, assim como Campsurus latipennis (Polymitarcyidae). Moradores locais as chamam vulgarmente de sarará (Figuras 1011) e citam grandes revoadas logo após os períodos chuvosos do ano. Primeiro registro para o Espírito Santo.

Material examinado: P54: (15A) 22.xi.2009.

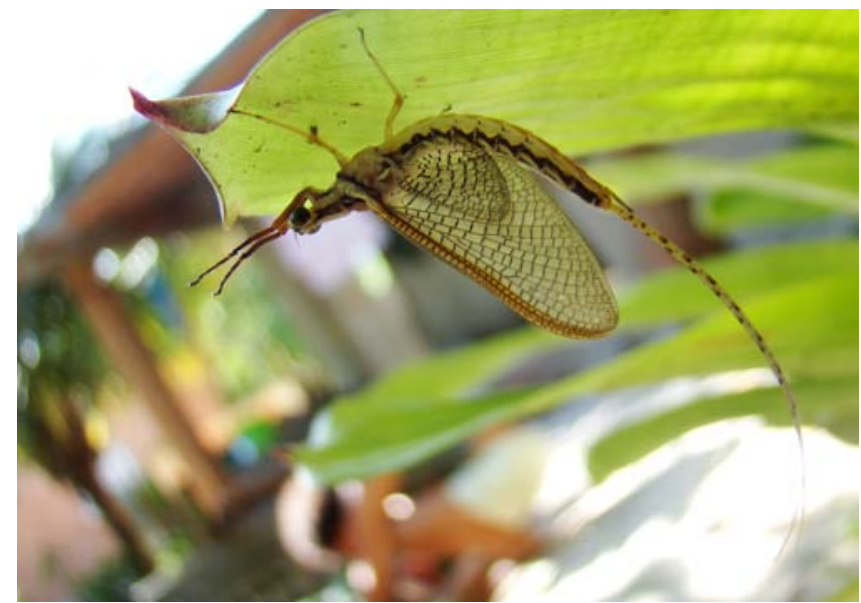

Figura 10. Subimago macho de Hexagenia (P.) albivitta (Walker, 1853) (Ephemeridae) coletada na Lagoa Juparanã, município de Sooretama, ES.

Figure 10. Male subimago of Hexagenia (P.) albivitta (Walker, 1853) (Ephemeridae) collected at Lagoa Juparanã municipality of Sooretama, ES.

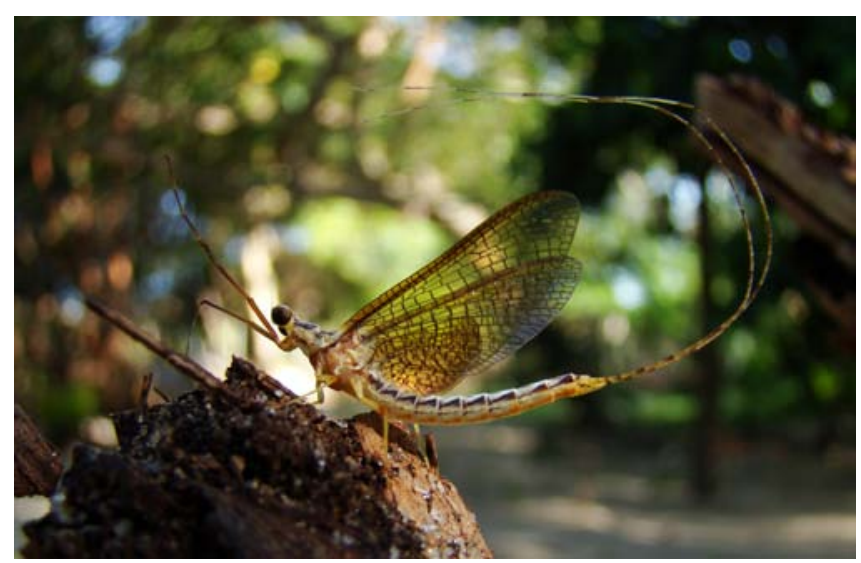

Figura 11. Imago macho de Hexagenia (P.) albivitta (Walker, 1853) (Ephemeridae) coletada na Lagoa Juparanã, município de Sooretama, ES.

Figure 11. Male imago Hexagenia (P.) albivitta (Walker, 1853) (Ephemeridae) collected at Lagoa Juparanã, municipality of Sooretama, ES.

\section{EUTHYPLOCIIDAE}

Campylocia anceps (Eaton, 1883)

Distribuição geográfica: América Central, Peru, Colômbia, Venezuela, Equador, Guiana Francesa, Guiana, Suriname, Brasil: Rio Grande do Sul, Amazonas, Pará, São Paulo, Rio de Janeiro, Espírito Santo (localidade desconhecida).

Comentários: Espécie previamente registrada para o estado (Ulmer 1942).

Campylocia dochmia Berner \& Thew, 1961

Distribuição geográfica: Brasil, Minas Gerais, Espírito Santo (Santa Teresa).

Comentários: Primeiro registro da espécie desde a sua descrição original, que tem sua localidade-tipo no Estado de São Paulo.

Material examinado: PT 45: (3A) 25.x.2008. 


\section{LEPTOHYPHIDAE}

Leptohyphes cornutus Allen, 1967

Distribuição geográfica: ARGENTINA, BRASIL: Santa Catarina, Goiás, Minas Gerais, Rio de Janeiro e Espírito Santo (Alegre).

Comentários: Espécie previamente refistrada para o estado (Dias et al. 2007), mas não encontrada durante o presente trabalho.

Leptohyphes plaumanni Allen, 1967

Distribuição geográfica: Argentina, Brasil: Santa Catarina, São Paulo, Minas Gerais, Rio de Janeiro e Espírito Santo (Alegre).

Comentários: Espécie previamente registradaa para o estado (Dias et al. 2007), mas não encontrada durante o presente trabalho.

Leptohyphodes inanis (Pictet, 1843)

Distribuição geográfica: Brasil: Minas Gerais, Rio de Janeiro,

São Paulo, Espírito Santo (Ibitirama, Santa Teresa).

Comentários: Primeiro registro para o Espírito Santo.

Material examinado: PT 19: (3N) 21.iv.2008; PT 21: (2N) 20.iv.2008; PT 22: (1N) 20.iv.2008; PT 44: (1N) 19.i.2008, (1N) 26.x.2008; PT 49: (2N) 20.ii.2009.

Traverhyphes (Mocoihyphes) yuati Molineri, 2004

Distribuição geográfica: Argentina, Brasil: São Paulo, Rio de Janeiro, Divisa Minas Gerais/Espírito Santo (Espera Feliz).

Comentários: Primeiro registro para o Espírito Santo.

Material examinado: PT 14: (2A) 25.iii.2009.

Traverhyphes (Traverhyphes) indicator (Needham \& Murphy, 1924)

Distribuição geográfica: Argentina, Uruguai, Brasil: São Paulo,

Espírito Santo (Pinheiros).

Comentários: Primeiro registro para o Espírito Santo.

Material examinado: PT 51: (4A) 26.x.2009.

Tricorythodes hiemalis Molineri, 2001

Distribuição geográfica: Argentina, Brasil: Mato Grosso, Espírito Santo (Alfredo Chaves, Ibitirama, Santa Teresa), Minas Gerais (Alto Caparaó), Divisa Minas Gerais/Espírito Santo (Espera Feliz).

Comentários: Primeiro registro para o Espírito Santo.

Material examinado: PT 04: (4N) 23.viii.2008; PT 08: (3N) 23.iii.2009; PT 11: (1N) 27.iii.2009; PT 19: (3N) 21.iv.2008; PT 21: (1N) 20.iv.2008; PT 22: (1N) 20.iv.2008; PT 44: (1N) 19.i.2008; PT 45: (1N) 20.i.2009; PT 45: (2N) 26.x.2008.

Tricorythodes mirca Molineri, 2002

Distribuição geográfica: Bolívia, Brasil: Espírito Santo (São Mateus, Sooretama).

Comentários: As ninfas examinadas não apresentavam dentículos submarginais nas garras tarsais, em contraste com os três apresentados na literatura para essa espécie (Molineri 2002, Dias et al. 2007). Consideramos, contudo, como uma variação específica. Primeiro registro para o Brasil.

Material examinado: PT 32: (7N) 30.i.2008; PT 37: (5N) 14.vii.2008; PT 37: (4N) 30.iv.2009; PT 38: (4N) 29.iv.2009; PT 41: (8N) 30.iv.2009; PT 42: (1N) 30.iv.2009; PT 42: (8N) 12.vii.2008; PT 42: (1N) 25.vii.2008.

Tricorythodes yura Molineri, 2002

Distribuição geográfica: Bolívia, Brasil: Espírito Santo (Santa Teresa, Sooretama).
Comentários: Primeiro registro para o Brasil.

Material examinado: PT 37: (3N) 14.vii.2008; PT 42: (3N) 15.vii.2008; PT 43: (1N) 25.x.2008; PT 43: (1N) 19.ii.2009; PT 44: (1N) 19.ii.2008; PT 44: (1N) 26.x.2008; PT 45: (1N) 19.i.2008; PT 45: (2N) 26.x.2008.

Tricorythodes sp.n.

Distribuição geográfica: BRASIL: Espírito Santo (Alfredo Chaves)

Material examinado: PT 04: (2N) 23.viii.2008.

Tricorythopsis artigas Traver, 1958

Distribuição geográfica: Argentina, Uruguai, Brasil: Rio de Janeiro, Rio Grande do Sul, São Paulo, Espírito Santo (Alfredo Chaves, Santa Teresa).

Comentários: Primeiro registro para o Espírito Santo.

Material examinado: PT 03: (7N) 24.vii.2008; PT 04: (21N) 23.viii.2008; PT 43: (3N) 20.i.2008; PT 43: (1N) 26.x.2008; PT 43:

(3N) 19.ii.2009; PT 43: (1N) 20.ii.2009; PT 47: (1N) 20.ii.2009.

Tricorythopsis araponga Dias \& Salles, 2005

Distribuição geográfica: Brasil: São Paulo, Minas Gerais, Rio de Janeiro e Espírito Santo (Jerônimo Monteiro).

Comentários: Espécie previamente registrada para o estado (Dias et al. 2007), mas não encontrada durante o presente trabalho.

Tricorythopsis pseudogibbus Dias \& Salles, 2005

Distribuição geográfica: Brasil: Minas Gerais, Rio de Janeiro e Espírito Santo (Alegre).

Comentários: Espécie previamente registrada para o estado (Dias et al. 2007), mas não encontrada durante o presente trabalho.

Tricorythopsis gibbus (Allen, 1967)

Distribuição geográfica: Argentina, Brasil: Rio de Janeiro, Santa Catarina, Espírito Santo (Santa Teresa).

Comentários: Primeiro registro para o Espírito Santo.

Material examinado: PT 43: (1N) 20.i.2008; PT 45: (3N)

19.i.2008; PT 45: (1N) 20.i.2008.

Tricorythopsis minimus (Allen, 1973)

Distribuição geográfica: Argentina, Uruguai, Brasil: Rio Grande do Sul, Espírito Santo (Sooretama).

Comentários: Primeiro registro para o Espírito Santo.

Material examinado: PT 37: (3N) 14.vii.2008.

Tricorythopsis undulatus (Allen, 1967)

Distribuição geográfica: Argentina, Brasil: Paraná, Espírito

Santo (Alfredo Chaves, Ibitirama).

Comentários: Primeiro registro para o Espírito Santo.

Material examinado: PT 04: (4N) 23.viii.2008; PT 09: (1N)

27.iii.2009; PT 22: (1N) 20.iv.2008

Tricorythopsis sp.n.

Distribuição geográfica: Brasil: Espírito Santo (Alfredo Chaves, Santa Teresa).

Material examinado: PT 04: (2N) 23.viii.2008; PT 43: (1N)

20.i.2008; PT 43: (1N) 25.x.2008; PT 44: (2N) 19.i.2008; PT 45: (5N) 20.i.2008; PT 45: (1N) 24.x.2008; PT 45: (1N) 26.x.2008; PT 47: (1N) 20.ii.2009.

\section{LEPTOPHLEBIIDAE}

Askola froehlichi Peters, 1969

Distribuição geográfica: Brasil: Rio Grande do Sul, Santa Catarina, Minas Gerais, Rio de Janeiro, São Paulo, Espírito Santo (Iúna, Santa Teresa) 
Comentários: Primeiro registro para o Espírito Santo.

Material examinado: PT 24: (1N) 21.iv.2008; PT 44: (1N) 19.ii.2008; PT 45: (1N) 21.iv.2008.

Farrodes carioca Dominguez, Molineri \& Peters, 1996

Distribuição geográfica: Brasil: Rio de Janeiro, Espírito Santo (Ibitirama, Santa Teresa, Sooretama)

Comentários: Primeiro registro para o Espírito Santo.

Material examinado: PT 19: (1N) 21.x.2008; PT 19: (1N) 26.x.2008; PT 19: (1N) 21.iv.2008; PT 23: (1N) 21.iv.2008; PT 37: (6N) 14.vii.2008; PT 37: (2N) 30.x.2008; PT 44: (12N) 16.xi.2007; PT 42: (7N) 15.vii.2008; PT 45: (1N) 18.i.2008; PT 45: (1A) 18.i.2008; PT 45: (22A) 20.i.2008; PT 45: (1N) 25.x.2008; PT 45: (1N) 26.x.2008; PT 45: (2A) 10.ii.2009.

Fittkaulus cururuensis Savage, 1986

Distribuição geográfica: Brasil: Pará, Espírito Santo (Sooretama).

Comentários: Espécie recentemente registrada para o estado (Boldrini et al. 2009).

Material examinado: PT 40: (16N) 15.vi.2008; PT 41: (1A) 14.ii.2008.

Hagenulopsis diptera Ulmer, 1920

Distribuição geográfica: Brasil: Santa Catarina, Espírito Santo (Ibitirama, Santa Teresa).

Comentários: Primeiro registro para o Espírito Santo.

Material examinado: PT 20: (1N) 20.iv.2008; PT 21: (1N) 20.iv.2008; PT 43: (1N) 25.x.2008; PT 43: (2N) 19.ii.2009; PT 45: (2N) 24.x.2008.

Hermanella froehlichi Ferreira \& Dominguez, 1992

Distribuição geográfica: Brasil: São Paulo, Espírito Santo (Ibitirama).

Comentários: Primeiro registro para o Espírito Santo.

Material examinado: PT 19: (1N) 21.iv.2008.

Hydrosmilodon gilliesae Thomas \& Péru, 2004

Distribuição geográfica: Guiana Francesa, Brasil: Espírito Santo (Sooretama).

Comentários: Primeiro registro da espécie para o Brasil.

Material examinado: PT 42: (12N) 15.vii.2008; PT 42: (1N) 02.iv.2009; PT 42: (4N) 29.iv.2009.

Hylister plaumanni Dominguez \& Flowers, 1989

Distribuição geográfica: Brasil: Paraná, Santa Catarina, Minas Gerais, Rio de Janeiro, Espírito Santo (Alfredo Chaves, Ibitirama, Iúna, Santa Teresa).

Comentários: Primeiro registro para o Espírito Santo.

Material examinado: PT 03: (7N) 24.vii.2008; PT 19: (4N) 21.iv.2008; PT 21: (10N) 20.iv.2008; PT 24: (5N) 23.viii.2008; PT 43: (1N) 20.i.2008; PT 43: (1N) 24.x.2008; PT 43: (1N) 25.x.2008; PT 44: (12N) 16.xi.2007; PT 44: (1A) 25.x.2008.

Massartella brieni (Lestage, 1924)

Distribuição geográfica: Brasil: Paraná, Rio Grande do Sul, Minas Gerais, Rio de Janeiro, São Paulo, Espírito Santo (Alto Caparaó, Ibitirma, Santa Teresa).

Comentários: Primeiro registro para o Espírito Santo.

Material examinado: PT 06: (1N) 23.iv.2008; PT 07: (1N) 23.iv.2008; PT 19: (2N) 21.iv.2008; PT 21: (1N) 20.iv.2008; PT 24: (12N) 21.iv.2008; PT 43: (1N) 19.ii.2009; PT 44: (23N) 16.xi.2007; PT 45: (3N) 26.x.2008; PT 45: (1A) 26.x.2008; PT 45: (4A) 20.i.2008; PT 46: (1N) 26.x.2008.
Miroculis mourei Savage \& Peters, 1983

Distribuição geográfica: Brasil: Paraná, Espírito Santo (Santa Teresa).

Comentários: Primeiro registro para o Espírito Santo.

Material examinado: PT 43: (3N) 18.i.2008; PT 43: (2N) 20.i.2008; PT 43: (1N) 24.x.2008; PT 45: (2N) 23.ii.2009; PT 48: (1N) 20.ii.2009.

Miroculis fittkaui Savage \& Peters, 1983

Distribuição geográfica: Suriname, Venezuela, Brasil: Pará, Espírito Santo (Sooretama).

Comentários: Primeiro registro para a Região Sudeste.

Material examinado: PT 39: (2A) 18.xii.2008.

Miroculis sp.n.

Distribuição geográfica: Brasil: Minas Gerais, Espírito Santo (Alto Caparaó, Ibitirama).

Material examinado: PT 05: (3N) 23.iv.2008; PT 19: (8N)

20.iv.2008; PT 19: (2N) 21.iv.2008; PT 22: (3N) 20.iv.2008.

Needhamella sp.n.

Distribuição geográfica: Brasil: Espírito Santo (Pinheiros).

Material examinado: PT 52: (1N) 26.x.2009; PT 52: (1A) 26.x.2009.

Paramaka sp.n.

Distribuição geográfica: Brasil: Espírito Santo (Alfredo Chaves, Ibitirama).

Material examinado: PT 04: (1N) 23.viii.2008; PT 21: (1N) 20.iv.2008.

Perissophlebiodes flinti Savage, 1982

Distribuição geográfica: Brasil: Bahia, São Paulo, Rio de Janeiro, Espírito Santo (Santa Teresa).

Comentários: Única espécie de Ephemeroptera presente na Lista das Espécies Brasileiras Ameaçadas de Extinção, (Ministério do Meio Ambiente 2008), foi encontrada apenas em uma propriedade particular no município de Santa Teresa. Primeiro registro para o Espírito Santo.

Material examinado: PT 44: (1N) 12.ii.2009; PT 44: (1N) 18.ii.2009; PT 45: (1N) 20.i.2008; PT 45: (5N) 25.x.2008; PT 45: (1N) 26.x.2008; PT 45: (2N) 20.ii.2009.

Simothraulopsis (Simothraulopsis) demerara (Traver, 1947)

Distribuição geográfica: Colômbia, Guiana Francesa, Suriname, Venezuela, Brasil: Amazonas, Pará, Rondônia, Espírito Santo (Alto Caparaó, Fundão, Ibitirama, Santa Teresa, São Mateus), Divisa Minas Gerais/Espírito Santo (Espera Feliz).

Comentários: Primeiro registro para a Região Sudeste.

Material examinado: PT 06: (1N) 25.iii.2009; PT 17: (1N) 23.iii.2009; PT 19: (1N) 21.iv.2008;PT 32: (41N) 27.x.2007; PT 44: (2N) 19.i.2008; PT 45: (2N) 18.i.2008; PT 45: (1A) 18.ii.2009; PT 47: (3N) 20.ii.2009. PT 50: (1N) 23.ii.2007.

Simothraulopsis (Maculoganthus) plesius Kluge, 2008

Distribuição geográfica: Peru, Brasil: Espírito Santo (Sooretama).

Comentários: Primeiro registro para o Brasil.

Material examinado: PT 40: (1N) 29.iv.2009; PT 40: (1N) 30.iv.2009.

Terpides sooretamae Boldrini \& Salles, 2009

Distribuição geográfica: Brasil: Mato Grosso, Espírito Santo (Alfredo Chaves, Sooretama, Pinheiros). 
Comentários: Espécie recentemente descrita com base em material da Reserva Biológica de Sooretama e do Mato Grosso (Boldrini et al. 2009).

Material examinado: PT 37: (1N) 30.iv.2009; PT 37: (1N) 15.vii.2008; PT 42: (3N) 30.iv.2009.

Thraulodes itatiajanus Traver \& Edmunds, 1967

Distribuição geográfica: Brasil: Rio de Janeiro, Espírito Santo

(Alto Caparaó, Ibitirama).

Comentários: Primeiro registro para o Espírito Santo.

Material examinado: PT 08: (4N) 23.iii.2009; PT 22: (3N) 20.iv.2008; PT 23: (7N) 21.iv.2008.

\section{Thraulodes sp. 1}

Distribuição geográfica: Brasil: Espírito Santo (Santa Teresa).

Comentários: Identificada com base em ninfas; sua associação aos adultos é imprescindível para que possa ser corretamente identificada.

Material examinado: PT 45: (10N) 21.x.2008; PT 45: (1N) 24.x.2008; PT 47: (5N) 25.x.2008.

\section{Thraulodes sp. 2}

Distribuição geográfica: Brasil: Espírito Santo (Santa Teresa).

Comentários: Identificada com base em ninfas; sua associação aos adultos é imprescindível para que possa ser corretamente identificada.

Material examinado: PT 45: (1N) 26.x.2008; PT 47: (12N) 20.i.2008; PT 47: (2N) 25.x.2008.

\section{Thraulodes sp. 3}

Distribuição geográfica: Brasil: Espírito Santo (Pinheiros).

Comentários: Identificada com base em ninfas; sua associação aos adultos é imprescindível para que possa ser corretamente identificada.

Material examinado: PT 52: (6N) 26.x.2009.

Ulmeritoides sp.n.

Distribuição geográfica: Brasil: Espírito Santo (Santa Teresa).

Material examinado: PT 45: (1N) 26.x.2008; PT 45: (1A) 26.x.2008; PT 47: (3N) 21.i.2008; PT 48: (1N) 20.ii.2009.

\section{MELANEMERELLIDAE}

Melanemerella brasiliana Ulmer, 1920

Distribuição geográfica: Brasil: Rio de Janeiro, São Paulo, Espírito Santo (município não informado).

Comentários: Espécie previamente registrada para o Estado (Ulmer 1920), mas não encontrada durante o presente trabalho. O Espírito Santo é a localidade-tipo da única espécie dessa família, porém apenas o nome do estado é mencionado na descrição original. Recentemente essa rara espécie de Ephemeroptera foi registrada para São Paulo e Rio de Janeiro, em áreas de altitude elevada (acima de 1500 metros) e vegetação do tipo Mata Atlântica bem preservada (Molineri \& Domínguez 2003).

\section{OLIGONEURIIDAE}

Lachlania sp.

Distribuição geográfica: BRASIL: Espírito Santo (Divisa ES/MG).

Comentários: Identificada com base em ninfas; sua associação aos adultos é imprescindível para que possa ser corretamente identificada.

Material examinado: P09: (5N) 24.iii.2009.

\section{POLYMITARCYIDAE}

Campsurus truncatus Ulmer, 1920

Distribuição geográfica: Bolívia; Brasil: Espírito Santo (Santa Teresa).

Comentários: Espécie previamente registrada para o estado (Ulmer 1920).

Material examinado: PT 44: (4A) 16.xi.2007.

Campsurus latipennis (Walker, 1853)

Distribuição geográfica: Brasil: Pará e Espírito Santo (Linhares e Sooretama).

Comentários: Espécie pela primeira vez registrada para a Região Sudeste. Apesar da identificação de muitas espécies de Campsurus Eaton ser comprometida em virtude das descrições originais antigas e incompletas, a genitália dos machos examinados está de acordo com a ilustração apresentada por Kimmins (1960) e realizada a partir do holótipo da espécie. Grandes revoadas dessa espécie são comuns na Lagoa Juparanã, onde os habitantes locais a chamam de sarará [em conjunto com Hexagenia (H.) albivitta].

Material examinado: P28: (50A) 12.ix.2009. 